\title{
Channel Abstractions for Network Security ${ }^{\dagger}$
}

\author{
MICHELE BUGLIESI, RICCARDO FOCARDI \\ Dipartimento di Informatica, Università Ca' Foscari Venezia. \\ Via Torino 155, 30172 Venezia-Mestre, Italy \\ Email: $\{$ bugliesi,focardi\}@dsi.unive.it
}

Received 21 January 2009; revised 5 October 2009

Process algebraic techniques for distributed systems are increasingly being targeted at identifying abstractions adequate both for high-level programming and specification, and for security analysis and verification. Drawing on our earlier work in (Bugliesi and Focardi, 2008), we investigate the expressive power of a core set of security and network abstractions that provide high-level primitives for the specifications of the honest principals in a network, while at the same time enabling an analysis of the network-level adversarial attacks that may be mounted by an intruder.

We analyze various bisimulation equivalences for security, arising from endowing the intruder with $(i)$ different adversarial capabilities and $(i i)$ increasingly powerful control on the interaction among the distributed principals of a network. By comparing the relative strength of the bisimulation equivalences, we obtain a direct measure of the discriminating power of the intruder, hence of the expressiveness of the corresponding intruder model.

\section{Introduction}

Achieving security in distributed systems is challenging and often creates a tension between two conflicting design requirements. On the one side, security requires formal, often low-level, specifications of the safeguards built against the threats to which the systems are exposed. On the other side, programming needs techniques and reasoning methods that abstract away from low-level details to focus instead on the expected functional properties.

In the literature on process calculi, this tension has generated a range of approaches, with two extremes. At one end, lie specifications based on the pi calculus (Milner et al., 1992). These assume very abstract mechanisms to secure communications by relying on private channels. While elegant and concise, the security guarantees conveyed by these mechanisms are often hard to realize in practice (Abadi, 1998). At the other end, we find specifications that draw on explicit cryptographic primitives as in the spi calculus (Abadi and Gordon, 1999) or in the applied-pi calculus (Abadi and Fournet, 2001). While this approach is very successful in providing a formal basis for the analysis of

\footnotetext{
$\dagger$ Work partially supported by M.I.U.R. (Italian Ministry of Education, University and Research) under project textitSOFT: Security-Oriented Formal Techniques
} 
network security applications, the resulting specifications are more naturally targeted at cryptographic protocols, and share the same limits of pi calculus when we try to hide the cryptographic layer and reason on secure communication in its essence.

Following a more recent line of research (Abadi and Fournet, 2004; Laud, 2005; Adão and Fournet, 2006; Corin et al., 2007; Fournet and Rezk, 2008), in our earlier work (Bugliesi and Focardi, 2008), we isolated a core set of security abstractions well-suited for high-level programming and system design, while at the same time amenable to distributed implementation and sufficiently expressive to represent full-blown adversarial settings. In the present paper, we further investigate the effectiveness of our approach by assessing the adequacy of our abstractions for security analysis. In particular, we analyze various bisimulation-based security equivalences for the calculus, associated with a variety of intruder models. The models arise from endowing the intruders with (i) different adversarial capabilities and (ii) increasingly powerful control on the interaction among the distributed principals of a network. The bisimulation equivalences, in turn, provide a direct measure of the discriminating power of the intruders, hence of the expressiveness of the corresponding models.

The starting point is the asynchronous pi-calculus with security abstractions we defined in (Bugliesi and Focardi, 2008) and the Dolev-Yao intruder model defined there. In this model, the intruder has the capability to interfere in all network interactions: it can forge its own traffic, intercept all messages and forward them back to the network, possibly replicating them. On the other hand, like the Dolev-Yao intruder studied in cryptographic protocols, it cannot learn any secret message and cannot forge any authenticated transmission. For this intruder model, we give a sound characterization of strong barbed equivalence in terms of strong asynchronous bisimulation. Also, we show that asynchronous and synchronous bisimilarity coincide.

We then extend our network abstractions with a new primitive that enables the intruder to silently eavesdrop on network traffic (without necessarily intercepting it). We show that the new capability adds no discriminating power to the intruder, in that it does not affect the security equivalences (either synchronous or asynchronous). On the other hand, eavesdropping turns out to be strictly less powerful than intercepting.

As a further step, we look at the notion of intruder adopted in (Adão and Fournet, 2006), that corresponds to what is sometimes referred to as the man-in-the-middle intruder. In this new model two principals may never engage in a synchronization directly as it is customary for the semantics of traditional process calculi (and as we assume in the initial model). Instead, all exchanges take place with the intruder's intervention. We show, somewhat surprisingly, that this additional control on the interactions on the network does not change the notion of equivalence, hence does not add discriminating power to the intruder.

Plan. Sections 2, 3 and 4 give an overview of the calculus and its semantics. Section 5 proves the main results on the security equivalence for the calculus and its coinductive characterization. Section 6 investigates alternative intruder models, and derives prowerful proof methods for bisimilarity. Section 7 discusses the import of such methods in the 
proofs of the distinctive equations for secrecy and authentication. Section 8 concludes the presentation.

The present paper revises and extends the results in (Bugliesi and Focardi, 2008) and (Bugliesi and Focardi, 2009).

\section{Security and Network Abstractions}

We start with a brief review of the calculus of security and network abstractions from (Bugliesi and Focardi, 2008). We presuppose two countable sets $\mathbf{N}$ and $\mathbf{V}$ of names and variables, respectively, and let $a-q$ range over names, $w, x, y, z$ over variables and $t, u, v$ over $\mathbf{N} \cup \mathbf{V}$. Names enable communication, but serve also as identities: for instance, $\bar{b}\langle a: \tilde{n}\rangle$ indicates an output to $b$ originating from $a$, while $b(a: \tilde{x})$ denotes an input performed by $b$ of a message from $a$. Tuples are indicated by a tilde, as in $\tilde{n}, \tilde{x}, \tilde{v}$.

\subsection{High-Level Principals}

The syntax of the high-level calculus is below.

\begin{tabular}{|c|c|c|}
\hline$H, K$ & $\begin{aligned}::= & \bar{u}\langle\underline{a}: \tilde{v}\rangle^{\circ} \\
& v(\underline{u}: \tilde{y})^{\circ} \cdot H \\
& \mathbf{0} \\
& H \mid K \\
& \text { if } u=v \text { then } H \text { else } K \\
& A\langle\tilde{u}\rangle \\
& (\boldsymbol{\nu} a) H\end{aligned}$ & $\begin{array}{l}\text { (Output) } \\
\text { (Input) } \\
\text { (Null) } \\
\text { (Parallel) } \\
\text { (Conditional) } \\
\text { (Definition) } \\
\text { (Restriction) }\end{array}$ \\
\hline
\end{tabular}

We use $\underline{u}$ as short for the name or variable $u$, or the distinguished name - associated with an anonymous identity. The notion of $\alpha$-renaming arises as expected. The null, parallel composition and conditional forms are just as in the pi-calculus. $A\langle\tilde{u}\rangle$ is the process defined by a (possibly recursive) definition $A(\tilde{x}) \stackrel{\text { def }}{=} H$, where $\tilde{x}$ contains all the variables that appear free in $H,|\tilde{u}|=|\tilde{x}|$, and $A$ may only occur guarded by an input prefix in $H$. The restriction $(\boldsymbol{\nu} a) H$ has the familiar pi-calculus syntax but weaker scoping rules to make it more adequate for implementation in distributed settings (see below). As to communication, we have four input/output forms, depending whether $\underline{a}$ is $a$ or and whether $\circ$ is $\bullet$ or the empty character.

The output forms are explained as follows: $\bar{u}\langle-: \tilde{v}\rangle$ denotes a plain output, a communication primitive that conveys no security guarantee; $\bar{u}\langle a: \tilde{v}\rangle$ denotes a public, but authentic output, which certifies the originator $a$ of the message, and ensures that the message cannot be replayed; notice that, in practice, replays are detected and discarded by the receiver via time-variant parameters included in the message, such as timestamps or nonces: the overall effect is that the message will be delivered once and, in our abstraction, this is modelled by directly forbidding replays of authenticated message. $\bar{u}\langle-: \tilde{v}\rangle^{\bullet}$ denotes a secret transmission, providing guarantees that only the intended receiver $u$ will have access to the message payload; finally, $\bar{u}\langle a: \tilde{v}\rangle \bullet$ denotes a secure transmission, combining the guarantees of the authentic and secret modes. In sum, the secret outputs 
protect from message disclosure, while authentic outputs protect against replication and forging. On the other hand, an opponent may intercept all outputs, and then selectively forward them back to the network.

The input forms have dual semantics: $v(\underline{u}: \tilde{y})^{\circ} . H$ denotes an input, which consumes a message sent on $v$ from $u$ or - , binding $\tilde{y}$ to the tuple of names that form the payload. The input prefix is thus a binder for the variable $\tilde{y}$, whose scope is $H$ : instead, $\underline{u}$ must be instantiated at the time the input prefix is ready to fire. As for output, o signals the secrecy mode and $\underline{u}$ the authenticity one. In the secret mode $v(\underline{u}: \tilde{y})^{\bullet} . H$ we always require that $v$ is a name. This is to avoid impersonation, as explained below. Inputs and outputs must agree on the transmission mode to synchronize.

Like in the local pi-calculus (Merro and Sangiorgi, 2004), we make a clear distinction between the input and output capabilities for communication, and we disallow the transmission of the former in the secret mode. Similarly, we require a name in the sender position of authentic messages. Taken together, these constraints guarantee that a process $H$ never gets to dynamically impersonate a new identity, i.e. use that identity as the subject of a secret input or, dually, as the source of an authentic output.

Definition 2.1 (Impersonation). A process $H$ impersonates an identity $a$ iff $a$ occurs free in $H$ either in the subject of a secret input, as in $a(\underline{u}: \tilde{y})^{\bullet} \cdot H^{\prime}$, or as the source of an authentic output, as in $\bar{u}\langle a: \tilde{v}\rangle^{\circ}$.

\subsection{Networks and Intruders}

Networks provide the low-level counterpart of the high-level calculus we just discussed. In addition, they make it possible to express the capabilities of an attacker. The syntax is given below: within networks, names are partitioned into two sets $\mathbf{N}_{t}$ and $\mathbf{N}_{u}$ of trusted and untrusted identities, respectively. By convention, we assume that $\alpha$-renaming respects this partition.

$$
\begin{array}{rlll}
M, N, O & := & \bar{u}\langle\underline{a}: \tilde{v} \| \tilde{t}\rangle^{\circ} & \text { (Low Output) } \\
& & v(\underline{u}: \tilde{y} \| \tilde{z})^{\circ} \cdot M & \text { (Low Input) } \\
& \mathbf{0}|M| N|A\langle\tilde{u}\rangle|(\boldsymbol{\nu} a) N \mid & \text { if } u=v \text { then } M \text { else } N \\
& \dagger z(x: \tilde{y} \| \tilde{w})_{i}^{\circ} \cdot M & \text { (Intercept) } \\
& \vdots i & \text { (Forward/Replay) }
\end{array}
$$

The first two productions introduce the network-level primitives for input and output and are subject to the same restrictions about the use of names as in the high-level syntax. The notion of impersonation carries over similarly, from high-level processes to networks, as expected. The novelty is in the additional components $\tilde{t}$ of the output messages: these represent (an abstraction of) the bitstring representation of the payload $\tilde{v}$, i.e. the view of the payload available to an external observer of the message, and are bound to the variables $\tilde{z}$ in the input prefix upon synchronization. The last two productions define the adversarial primitives. The intercept prefix $\dagger z(x: \tilde{y} \| \tilde{w})_{i}^{\circ} \cdot M$ enables an adversary to intercept all network messages. The prefix is a binder for the name $i$ and all its component variables, with scope $M$ : intercepting the output $\bar{b}\langle\underline{a}: \tilde{m} \| \tilde{n}\rangle^{\circ}$ creates a copy 
of the message indexed by the fresh name $i$ and binds $z$ to the target $b, x$ to $\underline{a}$ and $\tilde{w}$ to $\tilde{n}$. As to $\tilde{y}$, the binding depends on the secrecy mode of the message and on the trust status of the identity $b$. In particular, if the message is secret and $b \in \mathbf{N}_{t}$ then $\tilde{y}$ gets bound to $\tilde{n}$, otherwise $\tilde{y}$ is bound to $\tilde{m}$. Notice (i) that intercepting a secret message directed to a trusted principal does not break the secrecy of the payload, and (ii) that a message can be intercepted even if it is directed to a restricted identity, as in $(\boldsymbol{\nu} b) \bar{b}\langle\underline{a}: \tilde{m} \| \tilde{n}\rangle^{\circ}$. The indexed copies of the intercepted messages may be manipulated by way of the replay/forward form $! i$ that uses the index $i$ to forward a copy back to the network, or to produce a replica (in case the original messages was not authenticated).

We make the following, fairly mild, assumption on the format of messages in a network.

Definition 2.2 (Well-formed Networks). We say that a plain output $\bar{u}\langle-: \tilde{v} \| \tilde{t}\rangle$ is well-formed iff $\tilde{v}=\tilde{t}$; a secret/secure $\bar{u}\langle\underline{a}: \tilde{v} \| \tilde{t}\rangle^{\bullet}$ or authentic $\bar{u}\langle a: \tilde{v} \| \tilde{t}\rangle$ output is well-formed iff $|\tilde{v}|=|\tilde{t}|$. A network $N$ is well-formed iff it is closed (has no free variable) and all of its outputs are well-formed.

In other words, we assume that the two components $\tilde{v}$ and $\tilde{t}$ in all messages have the same arity, and that they coincide on public outputs. In fact, the bitstring of a message depends on the transmission mode: it coincides with the payload in plain outputs, while it is a fresh tuple of names in each authentic and/or secret output. For the trusted components of the network, the correspondence between message formats is established by the following translation of high-level principals $H$ into their network level counterparts $[H]$. We only give the clauses for the communication forms (the remaining clauses are defined homomorphically). As discussed in (Bugliesi and Focardi, 2008), in a cryptographic implementation, the chosen format may be realized by means of standard time-variant parameters as, e.g., timestamps, sequence numbers and nonces, in an authentic message, and by a randomized encryption in a secret output.

$$
\begin{aligned}
& {[\bar{u}\langle-: \tilde{v}\rangle] \quad \triangleq \bar{u}\langle-: \tilde{v} \| \tilde{v}\rangle} \\
& {[\bar{u}\langle a: \tilde{v}\rangle] \quad \triangleq(\boldsymbol{\nu} \tilde{c}) \bar{u}\langle a: \tilde{v} \| \tilde{c}\rangle \quad(|\tilde{v}|=|\tilde{c}|)} \\
& {\left[\bar{u}\langle\underline{a}: \tilde{v}\rangle^{\bullet}\right] \triangleq(\boldsymbol{\nu} \tilde{c}) \bar{u}\langle\underline{a}: \tilde{v} \| \tilde{c}\rangle^{\bullet} \quad(|\tilde{v}|=|\tilde{c}|)} \\
& {\left[b(\underline{u}: \tilde{x})^{\circ} \cdot H\right] \triangleq b(\underline{u}: \tilde{x} \| \tilde{y})^{\circ} \cdot[H] \quad(|\tilde{x}|=|\tilde{y}| \wedge \tilde{y} \cap f v(H)=\emptyset)}
\end{aligned}
$$

The partition on the set of identities in the two sets $\mathbf{N}_{t}$ and $\mathbf{N}_{u}$ makes it possible to identify, within a network, the trusted components from the intruder.

Definition 2.3 (Trusted processes vs Intruders). A network process $N$ is trusted iff $N=[H]$, for some high-level principal $H$, and $N$ only impersonates identities and creates fresh names in the set $\mathbf{N}_{t}$. A network process $N$ is an opponent/intruder iff it only impersonates identities and creates fresh names in the set $\mathbf{N}_{u}$

Throughout, we assume that our networks are well-formed and we reserve the letters $P$ and $Q$ to range over the class of trusted processes, and their run-time derivatives. 
Table 1 Structural Congruence and Reduction

Structural congruence. Let $T$ and $U$ range over high-level processes, or networks, uniformly in each of the following defining clauses.

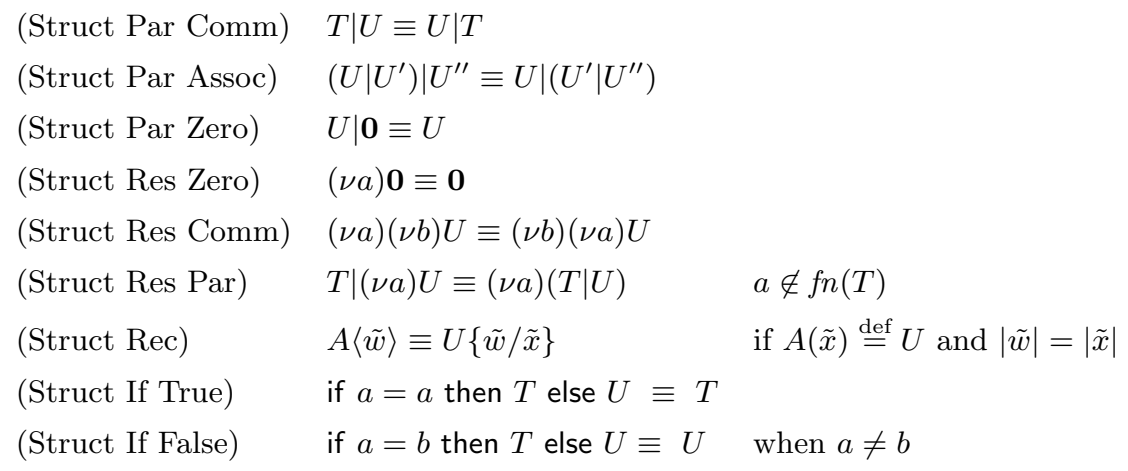

Reduction. In the (Intercept) rule $i \notin\{b, \underline{a}, \tilde{m}, \tilde{c}\}, \sigma$ is the substitution $\{b / z, \underline{a} / x, \tilde{p} / \tilde{y}, \tilde{c} / \tilde{w}\}$, and the $\tilde{p}$ are as follows: if $\circ=\bullet$ and $b \in \mathbf{N}_{t}$ then $\tilde{p}=\tilde{c}$ else $\tilde{p}=\tilde{m}$.

$$
\begin{aligned}
& \text { (Struct) } \\
& \frac{M \equiv M^{\prime} \quad M^{\prime} \longrightarrow N^{\prime} \quad N^{\prime} \equiv N}{M \longrightarrow N} \\
& \text { (Res) } \\
& \frac{N \longrightarrow N^{\prime}}{(\nu a) N \longrightarrow(\nu a) N^{\prime}} \quad \frac{M \longrightarrow M^{\prime}}{M\left|N \longrightarrow M^{\prime}\right| N} \\
& \text { (Intercept) } \quad \bar{b}\langle\underline{a}: \tilde{m} \| \tilde{c}\rangle^{\circ} \mid \dagger z(x: \tilde{y} \| \tilde{w})_{i}^{\circ} \cdot N \quad \longrightarrow \quad(\boldsymbol{\nu} i)\left(\bar{b}\langle\underline{a}: \tilde{m} \| \tilde{c}\rangle_{i}^{\circ} \mid N \sigma\right) \\
& (\mathrm{Comm}) \quad \bar{b}\langle\underline{a}: \tilde{m} \| \tilde{c}\rangle^{\circ} \mid b(\underline{a}: \tilde{y} \| \tilde{z})^{\circ} \cdot N \quad \longrightarrow \quad N\{\tilde{m} / \tilde{y}, \tilde{c} / \tilde{z}\} \\
& \text { (Forward) } \quad \bar{b}\langle a: \tilde{m} \| \tilde{c}\rangle_{i}^{\circ} \mid ! i \longrightarrow \bar{b}\langle a: \tilde{m} \| \tilde{c}\rangle^{\circ} \\
& \text { (Replay) } \quad \bar{b}\langle-: \tilde{m} \| \tilde{c}\rangle_{i}^{\circ}\left|! i \longrightarrow \bar{b}\langle-: \tilde{m} \| \tilde{c}\rangle_{i}^{\circ}\right| \bar{b}\langle-: \tilde{m} \| \tilde{c}\rangle^{\circ}
\end{aligned}
$$

\section{Reduction and Barbed Equivalence}

The dynamics of the calculus is given in Table 1, in terms of reduction and structural congruence. To formalize the dynamics of networks, we need a special form to represent the indexed copies of the messages stored upon interception. We introduce the new form below as part of what we call run-time network configurations:

$$
I, M, N \quad:=\ldots \text { as in Section } 2 \ldots \mid \bar{b}\langle\underline{a}: \tilde{m} \| \tilde{c}\rangle_{i}^{\circ}
$$

The index $i$ attached with an output is associated univocally with the intercept prefix that stored the indexed copy, as shown in the (Intercept) rule. Notice, in the same rule, that the bindings created depend on the structure and, more specifically, on the secrecy of the intercepted message, as explained earlier on. As for the remaining reductions, (Comm) is the usual synchronization rule, while (Forward) and (Reply) formalize the semantics of the adversarial form $! i$. Notice in particular that a non-authentic message is replicated, while an authentic one is not (the indexed copy is erased). 
The semantics of the calculus is completed by a notion of contextual equality based on reduction barbed congruence (Honda and Yoshida, 1995). We first define the observation predicate, as usual in terms of barbs.

Definition 3.1 (Barbs). We write $N \downarrow b$ whenever $N \equiv(\boldsymbol{\nu} \tilde{n})\left(\bar{b}\langle\ldots\rangle^{\circ} \mid N^{\prime}\right)$ and $b \notin \tilde{n}$

Definition 3.2 (Intruder Equivalence). A relation $\mathscr{R}$ on (run-time) networks is (i) barb preserving if $M \mathscr{R} N$ and $M \downarrow b$ imply $N \downarrow b$; (ii) reduction closed if $M \mathscr{R} N$ and $M \longrightarrow M^{\prime}$ imply $N \longrightarrow N^{\prime}$ with $M^{\prime} \mathscr{R} N^{\prime}$; (iii) contextual if $M \mathscr{R} N$ implies $M|I \mathscr{R} N| I$ for all intruder $I$ and $(\boldsymbol{\nu} \tilde{n}) M \mathscr{R}(\boldsymbol{\nu} \tilde{n}) N$ for all names $\tilde{n} \in \mathbf{N}$.

A symmetric relation is an intruder bisimulation if it is reduction closed, barb-preserving and contextual. Intruder equivalence, noted $\simeq$, is the largest intruder bisimulation.

Notice that we define our observation equivalence in terms of strong bisimulation, thus ending-up observing the silent moves of a process. However, since such moves arise from synchronizations over the network, this appears to be the only sound choice to make. Also we restrict to adversarial contexts, and define two processes equivalent if they cannot be distinguished by any opponent/intruder that observes them and/or actively interacts with them, reading, intercepting, forwarding and replaying the messages exchanged, or forging new ones. Indeed, this is a consequence of our initial intention, namely to find a reasoning method specifically targeted at the analysis of security-centric properties. On the other hand, the notion of equivalence we have adopted retains the expected congruence properties with respect to composition with trusted processes, subject to certain conditions on the identities such processes impersonate.

Theorem 3.1. Let $P, Q$ be trusted processes. $P \simeq Q$ implies $P|R \simeq Q| R$, for all trusted processes $R$ that do not impersonate any (trusted) identity in $f n(P, Q)$.

As a result, given $P, Q, R$ trusted, $P \simeq Q$ implies $P|R \simeq Q| R$ provided that the interactions between $P, Q$ and $R$ only occur via clear-text messages from $P$ (or $Q$ ) to $R$, and non-authentic messages from $R$ to $P(Q)$. Though they might at first appear overly restrictive, these constraints are indeed necessary conditions for the secure composition of trusted systems. To illustrate, given a trusted identity $b$, consider

$$
H(m)=\bar{b}\langle-: m\rangle^{\bullet} \mid b(-: x)^{\bullet} . S E A L\langle x\rangle .
$$

$H(m)$ is a secure principal that protects the secrecy of $m$ (as long as so does $S E A L\langle x\rangle$ ). This can be proved formally, showing that $[H(m)] \simeq\left[H\left(m^{\prime}\right)\right]$ for all pairs of $m$ and $m^{\prime}$ (see section 7). On the other hand, the security of $H$ is broken by any trusted process such as $R=b(-: x \| y)^{\bullet} \cdot \overline{l e a k}\langle-: x \| y\rangle$ that reads $x$ and then leaks it as a cleartext message: indeed $[H(m)]\left|R \not\left[H\left(m^{\prime}\right)\right]\right| R$ whenever $m \neq m^{\prime}$. The desired security guarantees may be recovered for $H$ by restricting the trusted identity $b$, so as to prevent $R$ from interfering on $b$. Indeed, $(\boldsymbol{\nu} b) H(m) \simeq(\boldsymbol{\nu} b) H\left(m^{\prime}\right)$ and this equality is preserved by the composition with any trusted process.

We give a formal proof of Theorem 3.1 in Section 5 after introducing the coinductive characterization of our observation equivalence in terms of labelled bisimilarity. Below, we illustrate the calculus and further elaborate on the security application of our notion 


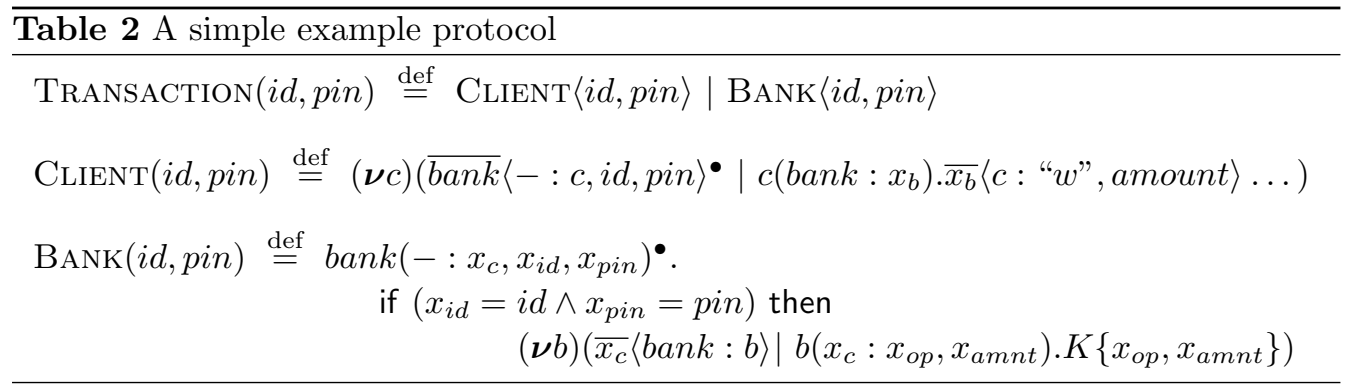

of equivalence and we put forward a simple e-banking protocol coded with our security abstractions.

\subsection{A simple on-line protocol}

The protocol involves two trusted principals, a BANK and a CLIENT, sharing information on the $i d$ and the pin of the client's account at the bank. The purpose of the protocol is for the client to withdraw a certain amount from his account. The interaction assumes that the BANK can be contacted at the publicly known identity bank $\in \mathbf{N}_{t}$, and takes the following three steps:

$$
\begin{aligned}
& \text { 1. Client } \stackrel{(\boldsymbol{\nu} c) \overline{b a n k}\langle-: c, i d, p i n\rangle^{\bullet}}{\longrightarrow} \text { BANK } \\
& \text { 2. Client } \longleftarrow(\boldsymbol{\nu} b) \bar{c}\langle\text { bank }: b\rangle \quad \text { BANK } \\
& \text { 3. Client } \longrightarrow \bar{b}\langle c: \text { : w", amount }\rangle \text { BANK }
\end{aligned}
$$

At step (1) CLIENT generates a fresh name $c$ to be used as his session identity, and communicates it to the bank together with the account $i d$ and pin. This communication is secret, to make sure that the account sensitive data is not leaked to any third party. At step (2) BANK responds with its own, freshly generated session identity $b$ : this communication is authentic, to protect $c$ against intruders trying to masquerade as the bank. Finally, at step (3) the client (more precisely, the instance of the client represented by the identity $c$ ) sends an order to withdraw amount to the bank (instance represented by $b$ ) terminating the protocol. The order request is authentic from $c$, to provide the bank with guarantees that the order has effectively originated from $c$ and is not a replica of a previous order.

The protocol and its participants are expressed directly in our calculus of high-level principals in terms of the definitions reported in Table 2. We can then formalize the main properties of the protocol, based on the notion of intruder equivalence introduced in this section. By an abuse of notation, we present the equations on the terms of the high-level calculus rather than on their corresponding network processes. In other words we write $H \simeq K$ as shorthand for $[H] \simeq[K]$. 
Client's account info is not leaked to any third party. One way to formalize this is by proving that no observer can distinguish two transactions based on the account data exchanged in the transaction, namely:

$$
\operatorname{Transaction}(i d, p i n) \simeq \operatorname{Transaction}\left(i d^{\prime}, p i n^{\prime}\right)
$$

Clearly, a proof of this equivalence requires that neither party deliberately leaks the account data once the transaction is complete. Another, more direct way that we can state that $i d$ and pin never reach an unintended recipient is by the following equivalence:

$$
\text { CLIENT }\langle i d, p i n\rangle \simeq(\boldsymbol{\nu} n) \overline{b a n k}\langle-: n, n, n\rangle^{\bullet}
$$

Here, we are equating CLIENT $\langle i d, p i n\rangle$ to a process that simply outputs a fresh name: that is exactly the view of the message output at the first protocol step available to the intruder, as the intruder cannot input on a trusted name such as bank. From this equation, we may also conclude that the protocol is resistant to attacks based on attempts to impersonate the bank, under the assumption the bank is completely off-line: notice, in fact, that there are no instances of the process BANK in parallel with the client. Since bank is a trusted name, no intruder will ever be able to forge the second protocol message, as it is authenticated.

BANK will only process orders originating from legitimate clients. This property can be expressed by the following equation,

$$
\operatorname{TransaCtion}(i d, p i n) \simeq \operatorname{TrANSACTION}_{\text {spec }}(i d, p i n)
$$

contrasting the formalization of the actual transaction with the formalization of an ideal transaction in which the two partners have previously agreed on the operation and the amount.

$$
\begin{aligned}
& \text { TrAnsaCtION }_{\text {spec }}(i d, p i n) \stackrel{\text { def }}{=} \operatorname{CLIENT}\langle i d, p i n\rangle \mid \mathrm{BANK}_{\text {spec }}\langle i d, p i n\rangle \\
& \mathrm{BANK}_{\text {spec }}(i d, p i n) \stackrel{\text { def }}{=} \operatorname{bank}\left(-: x_{c}, x_{i d}, x_{\text {pin }}\right)^{\bullet} . \\
& \text { if }\left(x_{i d}=i d \wedge x_{\text {pin }}=p i n\right) \text { then } \\
& (\boldsymbol{\nu} b)\left(\overline{x_{c}}\langle\text { bank }: b\rangle \mid b\left(x_{c}: x_{o p}, x_{a m n t}\right) \cdot K\{\text { "w", amount }\}\right)
\end{aligned}
$$

Notice, in fact, that the $\operatorname{BANK}_{s p e c}(i d, p i n)$ process calls $K\{$ “" $w$ ", amount $\}$ instead of the requested operation $K\left\{x_{o p}, x_{a m n t}\right\}$. If this specification is indistinguishable from the original process, we are guaranteed that no one can fool the bank into performing an operation different from the requested one.

\section{Labelled transitions}

We give an alternative formulation of the semantics of networks, based on a labelled transition system. The LTS is structured in two layers: the first layer, presented in this section, includes the transitions that match the reduction semantics of Section 3. A further layer, introduced in Section 5 will provide the basis for the definition of bisimilarity.

The first set of transitions is presented in Table 3. In most cases the transitions are 
Table 3 Labelled Transition Semantics

\section{Process and Intruder Transitions}

(Input)

(Output)

$b(\underline{a}: \tilde{y} \| \tilde{w})^{\circ} \cdot N \stackrel{b(\underline{a}: \tilde{m} \| \tilde{c})^{\circ}}{\longrightarrow} N\{\tilde{m} / \tilde{y}, \tilde{c} / \tilde{w}\}$

$\overline{\bar{b}\langle\underline{a}: \tilde{m} \| \tilde{c}\rangle^{\circ} \stackrel{\bar{b}\langle\underline{a}: \tilde{m} \| \tilde{c}\rangle^{\circ}}{\longrightarrow} \mathbf{0}}$

(Secret Output Intercepted)

$\frac{b \in \mathbf{N}_{t} \quad i \notin\{b, \underline{a}, \tilde{m}, \tilde{c}\}}{\bar{b}\langle\underline{a}: \tilde{m} \| \tilde{c}\rangle^{\bullet} \stackrel{(i) \dagger \bar{b}\langle\underline{a}: \tilde{c} \| \tilde{c}\rangle}{\longrightarrow} \bar{b}\langle\underline{a}: \tilde{m} \| \tilde{c}\rangle_{i}^{\bullet}}$

(Output Intercepted)

$\frac{b \notin \mathbf{N}_{t} \text { or } \circ \neq \bullet \quad i \notin\{b, \underline{a}, \tilde{m}, \tilde{c}\}}{\bar{b}\langle\underline{a}: \tilde{m} \| \tilde{c}\rangle^{\circ \stackrel{(i)+\bar{b}\langle\underline{a}: \tilde{m} \| \tilde{c}\rangle_{i}^{\circ}}{\longrightarrow}} \bar{b}\langle\underline{a}: \tilde{m} \| \tilde{c}\rangle_{i}^{\circ}}$

(Open)

(Open Intercepted)

$\frac{N \stackrel{(\tilde{p}) \bar{b}\langle\underline{a}: \tilde{m} \| \tilde{c}\rangle^{\circ}}{\longrightarrow} N^{\prime} \quad n \in\{\tilde{m}, \tilde{c}\} \backslash\{b, \underline{a}, \tilde{p}\}}{(\boldsymbol{\nu} n) N \stackrel{(n, \tilde{p}) \bar{b}\langle\underline{a}: \tilde{m} \| \tilde{c}\rangle^{\circ}}{\longrightarrow} N^{\prime}}$

$\frac{N \stackrel{(\tilde{p}, i) \dagger \bar{b}\langle\underline{a}: \tilde{m} \| \tilde{c}\rangle_{i}^{\circ}}{\longrightarrow} N^{\prime} n \in\{b, \underline{a}, \tilde{m}, \tilde{c}\} \backslash\{\tilde{p}, i\}}{(\boldsymbol{\nu} n) N \stackrel{(n, \tilde{p}, i) \dagger \bar{b}\langle\underline{a}: \tilde{m} \| \tilde{c}\rangle_{i}^{\circ}}{\longrightarrow} N^{\prime}}$

(Replay/Forward)

(Intercept)

$\stackrel{\stackrel{\langle i\rangle}{\longrightarrow} \mathbf{0}}{\mathbf{0}}$

$\frac{\sigma=\{b / z, \underline{a} / x, \tilde{p} / \tilde{y}, \tilde{c} / \tilde{w}\}}{\dagger z(x: \tilde{y} \| \tilde{w})_{i}^{\circ} \cdot N \stackrel{\dagger b(\underline{a}: \tilde{p} \| \tilde{c})_{i}^{\circ}}{\longrightarrow} N \sigma}$

(Restr)

$\frac{N \stackrel{\alpha}{\longrightarrow} N^{\prime} n \notin n(\alpha)}{(\boldsymbol{\nu} n) N \stackrel{\alpha}{\longrightarrow}(\boldsymbol{\nu} n) N^{\prime}}$

(Cond)

$\frac{(a=b \wedge M \stackrel{\alpha}{\rightarrow} N) \vee\left(a \neq b \wedge M^{\prime} \stackrel{\alpha}{\longrightarrow} N\right)}{\text { if } a=b \text { then } M \text { else } M^{\prime} \stackrel{\alpha}{\longrightarrow} N}$

(Par)

(Rec)

$\frac{M \stackrel{\alpha}{\longrightarrow} M^{\prime} \quad b n(\alpha) \cap f n(N)=\emptyset}{M|N, N| M \stackrel{\alpha}{\longrightarrow} M^{\prime}|N, N| M^{\prime}}$

$\frac{N\{\tilde{w} / \tilde{x}\} \stackrel{\alpha}{\longrightarrow} N^{\prime} A(\tilde{x}) \stackrel{\text { def }}{=} N}{A\langle\tilde{w}\rangle \stackrel{\alpha}{\longrightarrow} N^{\prime}}$

\section{Synchronization}

(Synch Intercept)

$$
\begin{aligned}
& M \stackrel{(\tilde{p}, i) \dagger \bar{b}\langle\underline{a}: \tilde{m} \| \tilde{c}\rangle_{i}^{\circ}}{\longrightarrow} M^{\prime} \quad N \stackrel{\dagger b(\underline{a}: \tilde{m} \| \tilde{c})_{i}^{\circ}}{\longrightarrow} N^{\prime} \quad\{\tilde{p}, i\} \cap f n(N)=\emptyset \\
& M \mid N \stackrel{\tau}{\longrightarrow}(\boldsymbol{\nu} \tilde{p}, i)\left(M^{\prime} \mid N^{\prime}\right)
\end{aligned}
$$

(Synch)

$\frac{M \stackrel{(\tilde{p}) \bar{b}\langle\underline{a}: \tilde{m} \| \tilde{c}\rangle^{\circ}}{\longrightarrow} M^{\prime} \quad N \stackrel{b(\underline{a}: \tilde{m} \| \tilde{c})^{\circ}}{\longrightarrow} N^{\prime} \quad \tilde{p} \cap f n(N)=\emptyset}{M \mid N \stackrel{\tau}{\longrightarrow}(\boldsymbol{\nu} \tilde{p})\left(M^{\prime} \mid N^{\prime}\right)}$

(Synch Index)

$$
\frac{M \stackrel{\langle i\rangle}{\longrightarrow} M^{\prime} N \stackrel{(i)}{\longrightarrow} N^{\prime}}{M\left|N \stackrel{\tau}{\longrightarrow} M^{\prime}\right| N^{\prime}}
$$

\section{Index transitions}

(Co-replay)

$\bar{b}\langle-: \tilde{m} \| \tilde{c}\rangle_{i}^{\circ} \stackrel{(i)}{\longrightarrow} \bar{b}\langle-: \tilde{m} \| \tilde{c}\rangle_{i}^{\circ} \mid \bar{b}\langle-: \tilde{m} \| \tilde{c}\rangle^{\circ}$
(Co-forward)

$\bar{b}\langle a: \tilde{m} \| \tilde{c}\rangle_{i}^{\circ} \stackrel{(i)}{\longrightarrow} \bar{b}\langle a: \tilde{m} \| \tilde{c}\rangle^{\circ}$ 
either standard, or constitute the direct counterpart of the corresponding reductions in Table 1. The two (Output Intercepted) transitions deserve more attention. First notice that, when the receiver is trusted, the label exhibits different information depending on the secrecy mode of the output. Secondly, observe that the transitions leave in their residual a indexed copy of the message emitted: this reflects the effect of an interaction with a surrounding context that tests the presence of an output by intercepting it. A further remark is in order on the difference between the two rules that govern scope extrusion. The difference is best understood if we take the view that a channel name comprises the two identities of the end-points it connects: the source and the destination. Under this interpretation the (Open) rule states that the channel name is not extruded, as in the pi-calculus, while the (Open Intercepted) opens the scope in accordance with the reduction semantics, by which intercepting a message discloses the identity of the receiver (as well of the sender) even though restricted. The following, standard result connects the reductions with the silent actions in the labelled transition semantics.

\section{Lemma 4.1 (Harmony).}

— If $M \stackrel{\alpha}{\longrightarrow} M^{\prime}$ and $M \equiv N$ then $N \stackrel{\alpha}{\longrightarrow} N^{\prime}$ and $M^{\prime} \equiv N^{\prime}$

$-N \longrightarrow N^{\prime}$ if and only if $N \stackrel{\tau}{\longrightarrow} \equiv N^{\prime}$.

We introduce an important class of processes, those arising from the trusted processes of Definition 2.3 by the labelled transitions we just introduced.

Definition 4.1 (Trusted Derivatives). A trusted derivative is a process obtained by a (possibly empty) sequence of labelled transitions from a trusted process. Inductively, $P$ is a trusted derivative if either $P \equiv[H]$ for some high-level principal $H$, or $\hat{P} \stackrel{\alpha}{\longrightarrow} P$ with $\hat{P}$ trusted derivative.

We prove a preliminary, but important lemma that characterizes various useful properties on the structure of trusted derivatives. We first introduce the following notation to help formalize such properties.

We write $P\rfloor_{\tilde{c}}$ whenever $P$ has an unguarded (indexed) output with a free bitstring $\tilde{c}$, and the output is either authentic and/or encrypted. Similarly, we write $P l_{i}$ to signal that $P$ has an indexed output with free index $i$. Formally:

$$
\begin{aligned}
P\rfloor_{\tilde{c}} \triangleq & P \text { is structurally congruent to any of the processes }(\boldsymbol{\nu} \tilde{p})\left(\hat{P} \mid \bar{b}\langle a: \tilde{m} \| \tilde{c}\rangle^{\circ}\right), \\
& (\boldsymbol{\nu} \tilde{p})\left(\hat{P} \mid \bar{b}\langle a: \tilde{m} \| \tilde{c}\rangle_{i}^{\circ}\right),(\boldsymbol{\nu} \tilde{p})\left(\hat{P} \mid \bar{b}\langle-: \tilde{m} \| \tilde{c}\rangle^{\bullet}\right), \text { or }(\boldsymbol{\nu} \tilde{p})\left(\hat{P} \mid \bar{b}\langle-: \tilde{m} \| \tilde{c}\rangle_{i}^{\bullet}\right) \\
& \text { with } \tilde{c} \cap \tilde{p}=\emptyset . \\
P l_{i} \triangleq & P \equiv(\boldsymbol{\nu} \tilde{p})\left(\hat{P} \mid \bar{b}\langle\underline{a}: \tilde{m} \| \tilde{c}\rangle_{i}^{\circ}\right) \text { with } i \notin \tilde{p} .
\end{aligned}
$$

Now item 1, in Lemma 4.2 below, states that the index of any output occurring in a trusted derivative is always free, as are the relative $b, \underline{a}$ and $\tilde{c}$, as all of these values have been intercepted. If the secrecy mode is plain, then even the payload $\tilde{m}$ is free. Item 2 states that the index is unique. Item 3 states that each bitstring $\tilde{c}$ identifies one and just one authentic output. This is not true for non-authentic messages as they could have been replicated. 
Lemma 4.2 (Properties of trusted derivatives). Let $P$ be a trusted derivative.

1 If $P \equiv(\boldsymbol{\nu} \tilde{p})\left(\hat{P} \mid \bar{b}\langle\underline{a}: \tilde{m} \| \tilde{c}\rangle_{i}^{\circ}\right)$ then $\{i, b, \underline{a}, \tilde{c}\} \cap\{\tilde{p}\}=\emptyset$. Furthermore if $\circ=\varepsilon$ or $b \in \mathbf{N}_{u}$, then $\{\tilde{m}\} \cap\{\tilde{p}\}=\emptyset$.

2 If $P \equiv(\boldsymbol{\nu} \tilde{p})\left(\hat{P} \mid \bar{b}\langle\underline{a}: \tilde{m} \| \tilde{c}\rangle_{i}^{\circ}\right)$ then $\hat{P} \chi_{i}$.

3 If $P \equiv(\boldsymbol{\nu} \tilde{p})\left(\hat{P} \mid \bar{b}\langle a: \tilde{m} \| \tilde{c}\rangle^{\circ}\right)$ then $\hat{P} \chi_{\tilde{c}}$.

Proof. We prove each of the items in turn: in all cases, the proof is by induction, based on the inductive definition of trusted derivative.

Proof of (1). In the base case the claim follows vacuously, as trusted processes do not have any occurrence of indexed outputs. For the inductive case, assume the claim is true for the trusted derivative $P$, and let $P \stackrel{\alpha}{\longrightarrow} P^{\prime}$. We need to show that the desired property holds of $(i)$ all the indexed outputs occurring in $P$ that outlive the transition and are thus found back in $P^{\prime}$; and of $(i i)$ any new indexed output generated by the transition itself.

For $(i)$ it is enough to observe that no name free in $P$ may ever get bound in $P^{\prime}$, simply because no labelled transition introduces new binders. As to $(i i)$ if the transition generates a new indexed output, then it must be of the form

$$
P \equiv(\boldsymbol{\nu} \tilde{p}, \tilde{r})\left(P^{*} \mid \overline{b^{\prime}}\left\langle\underline{a}^{\prime}: \tilde{m}^{\prime} \| \tilde{c}^{\prime}\right\rangle^{\circ}\right) \stackrel{(\tilde{r}, j)+\overline{b^{\prime}}\left\langle\underline{a}^{\prime}: \tilde{n} \| \tilde{c}^{\prime}\right\rangle_{j}^{\circ}}{\longrightarrow} P^{\prime} \equiv(\boldsymbol{\nu} \tilde{p})\left(P^{*} \mid \overline{b^{\prime}}\left\langle\underline{a}^{\prime}: \tilde{m}^{\prime} \| \tilde{c}^{\prime}\right\rangle_{j}^{\circ}\right)
$$

where $\tilde{n}=\tilde{m}^{\prime}$ if $b^{\prime} \notin \mathbf{N}_{t}$ or $\circ \neq \bullet$, and $\tilde{n}=\tilde{c}^{\prime}$ otherwise. The side conditions to the (Restr) and (Open Intercept) rules enforce the conditions required by the Lemma on the output indexed by $j$.

Proof of (2). As in item (1), the base case follows vacuously. For the inductive case, assume the desired property holds of $P$, and consider the new trusted derivative obtained by $P \stackrel{\alpha}{\longrightarrow} P^{\prime}$. If $\alpha$ does not generate a new indexed output, the lemma follows directly by the induction hypothesis, because $\left.P^{\prime}\right|_{i}$ implies $P l_{i}$ for all indexes $i$. Otherwise, as in the previous case, the transition has the form

$$
P \equiv(\boldsymbol{\nu} \tilde{p}, \tilde{r})\left(P^{*} \mid \overline{b^{\prime}}\left\langle\underline{a}^{\prime}: \tilde{m}^{\prime} \| \tilde{c}^{\prime}\right\rangle^{\circ}\right) \stackrel{(\tilde{r}, j)+\overline{b^{\prime}}\left\langle\underline{a}^{\prime}: \tilde{n} \| \tilde{c}^{\prime}\right\rangle_{j}^{\circ}}{\longrightarrow} P^{\prime} \equiv(\boldsymbol{\nu} \tilde{p})\left(P^{*} \mid \overline{b^{\prime}}\left\langle\underline{a}^{\prime}: \tilde{m}^{\prime} \| \tilde{c}^{\prime}\right\rangle_{j}^{\circ}\right)
$$

To conclude, we need to show that $j \neq i$ for all $i$ such that $P{ }_{i}$. From item (1) of the present lemma, we know that $i \in f n(P)$, hence $i \in f n\left(P^{*}\right)$ Then, $j \neq i$ follows by the side condition that governs the choice of the bound names in rule (Par).

Proof of (3). We need a more general statement to carry out the inductive proof, namely: For all $\tilde{c}$ such that $P \equiv(\boldsymbol{\nu} \tilde{p})\left(\hat{P} \mid \bar{b}\langle a: \tilde{m} \| \tilde{c}\rangle^{\circ}\right)$ or $P \equiv(\boldsymbol{\nu} \tilde{p})\left(\hat{P} \mid \bar{b}\langle a: \tilde{m} \| \tilde{c}\rangle_{i}^{\circ}\right)$ one has $\hat{P} \chi_{\tilde{c}}$.

In the base case, $P \equiv[H]$ has no indexed outputs. As to non-indexed outputs, an analysis of the translation [.] shows that $P$ may be restructured as $(\boldsymbol{\nu} \tilde{r})\left(\hat{P} \mid(\boldsymbol{\nu} \tilde{c}) \bar{b}\langle a: \tilde{m} \| \tilde{c}\rangle^{\circ}\right)$, where $\tilde{p}=\tilde{r} \uplus \tilde{c}$ : from this, it is clear that $\hat{P} \chi_{\tilde{c}}$, as desired. In the inductive case, assume the claim true of a trusted derivative $P^{*}$ and let $P^{*} \stackrel{\alpha}{\longrightarrow} P$. We reason by cases on the format of $\alpha$. 
If $\alpha$ is an input, then $P^{*} \equiv(\boldsymbol{\nu} \tilde{p})\left(\hat{P}^{*} \mid d(\underline{e}: \tilde{x} \| \tilde{y}) \cdot[H]\right)$ and $P \equiv(\boldsymbol{\nu} \tilde{p})\left(\hat{P}^{*} \mid[H \sigma]\right)$ for a suitable substitution $\sigma$ of the variables in $\tilde{x}$ and $\tilde{y}$. Now, for all the unguarded outputs in $[H \sigma]$ we reason as in the based case. For the remaining (indexed) outputs in $\hat{P}^{*}$ the claim follows by the induction hypothesis.

If $\alpha$ is an output/intercept/co-forward, the proof follows directly by the induction hypothesis. For the output case, all the (indexed and not) unguarded outputs of $P$ are unguarded in $P^{*}$; for the intercept case, $P^{*} \equiv(\boldsymbol{\nu} \tilde{p})\left(\hat{P}^{*} \mid \bar{b}\langle a: \tilde{m} \| \tilde{c}\rangle^{\circ}\right)$ and $P \equiv$ $(\boldsymbol{\nu} \tilde{p})\left(\hat{P}^{*} \mid \bar{b}\langle a: \tilde{m} \| \tilde{c}\rangle_{i}^{\circ}\right)$ and $\hat{P}^{*} \chi_{\tilde{c}}$ is implied by the induction hypothesis on $P^{*}$. For the co-forward case, the reasoning is the same as the one just described, with $P \equiv$ $(\boldsymbol{\nu} \tilde{p})\left(\hat{P}^{*} \mid \bar{b}\langle a: \tilde{m} \| \tilde{c}\rangle^{\circ}\right)$ and $P^{*} \equiv(\boldsymbol{\nu} \tilde{p})\left(\hat{P}^{*} \mid \bar{b}\langle a: \tilde{m} \| \tilde{c}\rangle_{i}^{\circ}\right)$.

\section{Bisimilarity}

As anticipated, the definition of bisimilarity rests on a further set of labelled transitions, that provide the observable counterpart of the labelled transitions of Table 3 . The new transitions are introduced in Definition 5.1 below, and are obtained from the transitions in in Table 3 by filtering away all the transitions that involve the adversarial forms (intercept and forward/reply) as well all the transitions that may not be observed by an opponent by virtue of the restriction the opponent suffers on the use of the trusted identities of a network.

Definition 5.1 (Observable LTS). We say that a network has an observable transition, noted $N \stackrel{\alpha}{\longmapsto} N^{\prime}$, if and only if it may be derived by the following rules:

$$
\frac{N \stackrel{\alpha}{\longrightarrow} N^{\prime}}{N \stackrel{\alpha}{\longmapsto} N^{\prime}} \quad \alpha \notin\left\{\begin{array}{ll}
b(a: \tilde{m} \| \tilde{c})^{\circ} & a \in \mathbf{N}_{t} \\
(\tilde{p}) \bar{b}\left\langle\underline{a}: \tilde{m} \| \tilde{c} \bullet^{\bullet}\right. & b \in \mathbf{N}_{t} \\
\dagger b(\underline{a}: \tilde{m} \| \tilde{c})_{i}^{\circ},\langle i\rangle &
\end{array}\right\}
$$

The notions of synchronous and asynchronous bisimulation arise as expected from the observable labelled transitions. When $\alpha$ is an input action, say $b(\underline{a}: \tilde{m} \| \tilde{c})^{\circ}$, we note with $\bar{\alpha}$ the corresponding output action $\bar{b}\langle\underline{a}: \tilde{m} \| \tilde{c}\rangle^{\circ}$.

Definition 5.2 (Intruder Bisimularity). Let $\mathscr{R}$ be a symmetric relation over networks. $\mathscr{R}$ is a bisimulation if whenever $M \mathscr{R} N$ and $M \stackrel{\alpha}{\longmapsto} M^{\prime}$ with $b n(\alpha) \cap f n(N)=\emptyset$ there exists $N^{\prime}$ such that $N \stackrel{\alpha}{\longmapsto} N^{\prime}$ and $M^{\prime} \mathscr{R} N^{\prime}$.

$\mathscr{R}$ is an asynchronous bisimulation if whenever $M \mathscr{R} N$ and $M \stackrel{\alpha}{\longmapsto} M^{\prime}$ with $b n(\alpha) \cap$ $f n(N)=\emptyset$ one has: (i) if $\alpha$ is not an input, then $N \stackrel{\alpha}{\longmapsto} N^{\prime}$ and $M^{\prime} \mathscr{R} N^{\prime}$; (ii) if $\alpha$ is an input, then $N \stackrel{\alpha}{\longmapsto} N^{\prime}$ and $M^{\prime} \mathscr{R} N^{\prime}$ or $N \stackrel{\tau}{\longmapsto} N^{\prime}$ and $M^{\prime} \mathscr{R} N^{\prime} \mid \bar{\alpha}$.

Bisimilarity, noted $\sim$, is the largest bisimulation, and asynchronous bisimilarity, noted $\sim_{a}$, is the largest asynchronous bisimulation.

In the proofs, it will be convenient to work with bisimulations up to structural congruence, i.e., bisimulations in which matching actions lead to processes which are still in $\mathscr{R}$ up to $\equiv$. In particular, the requirement $M^{\prime} \mathscr{R} N^{\prime}\left(M^{\prime} \mathscr{R} N^{\prime} \mid \bar{\alpha}\right.$, for the asynchronous input case) is relaxed into $M^{\prime} \equiv \mathscr{R} \equiv N^{\prime}\left(M^{\prime} \equiv \mathscr{R} \equiv N^{\prime} \mid \bar{\alpha}\right.$, respectively $)$. Thanks to Lemma 4.1, it is trivial 
to prove that that if $\mathscr{R}$ is a (asynchronous) bisimulation up to structural congruence then $\equiv \mathscr{R} \equiv$ is a (asynchronous) bisimulation. Thus, in order to prove that two processes are bisimilar it is sufficient to exhibit a bisimulation up to structural congruence containing them. In the following, we will implicitly adopt this technique.

\subsection{Synchronous vs asynchronous bisimilarity}

Given the asynchronous nature of the calculus, it would seem natural to elect $\sim_{a}$ as the natural bisimilarity. As it turns out, however, the ability to intercept all traffic makes asynchronous bisimilarity just as powerful as synchronous bisimilarity. We prove this below. To ease the presentation and the proofs, we tacitly adopt the so-called Barendregt convention for the bound and free names of a process: in particular, we assume bound names to be all distinct and different from the free names of all the considered processes. One consequence of this convention is that we may simplify the definition of bisimilarity by dropping the side-condition " $b n(\alpha) \cap f n(N)=\emptyset$ " as it is verified trivially by virtue of the convention.

We first prove two simple, but useful, lemmas.

Lemma 5.1. Let $\bar{\gamma}_{i}=\bar{a}\langle\underline{b}: \tilde{m} \| \tilde{c}\rangle_{i}^{\circ}$ and $\bar{\gamma}_{i}^{\prime}=\bar{a}^{\prime}\left\langle\underline{b}^{\prime}: \tilde{m}^{\prime} \| \tilde{c}^{\prime}\right\rangle_{i}^{\circ}$. If $(\boldsymbol{\nu} \tilde{p})\left(P \mid \bar{\gamma}_{i}\right) \frown(\boldsymbol{\nu} \tilde{q})\left(Q \mid \bar{\gamma}_{i}^{\prime}\right)$ then also $(\boldsymbol{\nu} \tilde{p}) P \frown(\boldsymbol{\nu} \tilde{q}) Q$, where $\frown$ is either $\sim$ or $\sim_{a}$, respectively.

Proof. Define $\mathscr{R}=\left\{((\boldsymbol{\nu} \tilde{p}) P,(\boldsymbol{\nu} \tilde{q}) Q) \mid(\boldsymbol{\nu} \tilde{p})\left(P \mid \bar{\gamma}_{i}\right) \frown(\boldsymbol{\nu} \tilde{q})\left(Q \mid \bar{\gamma}_{i}^{\prime}\right)\right\}$ : we show that $\mathscr{R}$ is a $\frown$-bisimulation (up to structural congruence). Let $(\boldsymbol{\nu} \tilde{p}) P \mathscr{R}(\boldsymbol{\nu} \tilde{q}) Q$ and assume that $(\boldsymbol{\nu} \tilde{p}) P \stackrel{\alpha}{\longmapsto}\left(\boldsymbol{\nu} \tilde{p}^{\prime}\right) P^{\prime}$. Then also $P \stackrel{\hat{\alpha}}{\longmapsto} P^{\prime}$ for a some $\hat{\alpha}$ : in particular, either $\alpha=(\tilde{r}) \hat{\alpha}$ and $\tilde{p}=\left\{\tilde{p}^{\prime}, \tilde{r}\right\}$, or $\alpha=\hat{\alpha}$ and $\tilde{p}=\tilde{p}^{\prime}$. By Lemma 4.2(2), we know that $\hat{\alpha} \neq(i)$, hence $\alpha \neq(i)$. By the Barendregt convention, we also have that $P\left|\bar{\gamma}_{i} \stackrel{\hat{\alpha}}{\longmapsto} P^{\prime}\right| \bar{\gamma}_{i}$. Consequently, $(\boldsymbol{\nu} \tilde{p})\left(P \mid \bar{\gamma}_{i}\right) \stackrel{\alpha}{\longmapsto}\left(\boldsymbol{\nu} \tilde{p}^{\prime}\right)\left(P^{\prime} \mid \bar{\gamma}_{i}\right)$. Now we may use the hypothesis $(\boldsymbol{\nu} \tilde{p})\left(P \mid \bar{\gamma}_{i}\right) \frown(\boldsymbol{\nu} \tilde{q})\left(Q \mid \bar{\gamma}_{i}^{\prime}\right)$ to find a matching transition from $(\boldsymbol{\nu} \tilde{q}) Q$. We have two cases.

If $\frown$ is $\sim$ or $\alpha$ is not an input action, we know that $(\boldsymbol{\nu} \tilde{q})\left(Q \mid \bar{\gamma}_{i}^{\prime}\right) \stackrel{\alpha}{\longmapsto}\left(\boldsymbol{\nu} \tilde{q}^{\prime}\right) R$ with $\left(\boldsymbol{\nu} \tilde{p}^{\prime}\right)\left(P^{\prime} \mid \bar{\gamma}_{i}\right) \frown\left(\boldsymbol{\nu} \tilde{q}^{\prime}\right) R$ and to conclude we must show that $(\boldsymbol{\nu} \tilde{q}) Q \stackrel{\alpha}{\longmapsto}\left(\boldsymbol{\nu} \tilde{q}^{\prime}\right) Q^{\prime}$ and that $R=Q^{\prime} \mid \bar{\gamma}_{i}^{\prime}$. Indeed, both these facts follow from the observation that $\alpha \neq(i)$ and that the only action performed by $\bar{\gamma}_{i}^{\prime}$ is $(i)$.

If instead $\alpha$ is an input action and $\frown$ is $\sim_{a}$, then we have an additional case, namely $(\boldsymbol{\nu} \tilde{q})\left(Q \mid \bar{\gamma}_{i}^{\prime}\right) \stackrel{\tau}{\longmapsto}\left(\boldsymbol{\nu} \tilde{q}^{\prime}\right) R$ with $\left(\boldsymbol{\nu} \tilde{p}^{\prime}\right)\left(P^{\prime} \mid \bar{\gamma}_{i}\right) \sim_{a}\left(\boldsymbol{\nu} \tilde{q}^{\prime}\right) R \mid \bar{\alpha}$. Reasoning as above, it follows that $R=Q^{\prime} \mid \bar{\gamma}_{i}^{\prime}$, and $(\boldsymbol{\nu} \tilde{q}) Q \stackrel{\tau}{\longmapsto}\left(\boldsymbol{\nu} \tilde{q}^{\prime}\right) Q^{\prime}$ which is again the matching transition we are looking for. In fact, we have $\left(\boldsymbol{\nu} \tilde{p}^{\prime}\right)\left(P^{\prime} \mid \bar{\gamma}_{i}\right) \sim_{a}\left(\boldsymbol{\nu} \tilde{q}^{\prime}\right) R \mid \bar{\alpha} \equiv\left(\boldsymbol{\nu} \tilde{q}^{\prime}\right)\left(Q^{\prime}|\bar{\alpha}| \bar{\gamma}_{i}^{\prime}\right)$ from which $\left(\boldsymbol{\nu} \tilde{p}^{\prime}\right) P^{\prime} \mathscr{R}\left(\boldsymbol{\nu} \tilde{q}^{\prime}\right)\left(Q^{\prime} \mid \bar{\alpha}\right) \equiv\left(\boldsymbol{\nu} \tilde{q}^{\prime}\right) Q^{\prime} \mid \bar{\alpha}$ as desired.

Lemma 5.2. If $P \frown Q$ then $(\boldsymbol{\nu} n) P \frown(\boldsymbol{\nu} n) Q$, where $\frown$ is either $\sim$ or $\sim_{a}$, respectively.

Proof. Directly, by coinduction.

Theorem 5.1. $\sim_{a}=\sim$.

Proof. Clearly $\sim \subseteq \sim_{a}$ because, by definition, a synchronous bisimulation is also an 
asynchronous bisimulation. To prove the reverse inclusion, let $\mathscr{R}=\left\{(P, Q) \mid P \sim_{a} Q\right\}$ : we show that $\mathscr{R}$ is a synchronous bisimulation.

Let $(P, Q) \in \mathscr{R}$ and $P \stackrel{\beta}{\longmapsto} P^{\prime}$. If $\beta$ is not an input action the proof follows directly from the hypothesis. In fact, since $P \sim_{a} Q$ by hypothesis, there exists a matching transition $Q \stackrel{\beta}{\longmapsto} Q^{\prime}$ such that $P^{\prime} \sim_{a} Q^{\prime}$. Hence $\left(P^{\prime}, Q^{\prime}\right) \in \mathscr{R}$ as desired.

Assume then that $\beta$ is an input action: $\beta=b(\underline{a}: \tilde{m} \| \tilde{c})^{\circ}$. Given that $P \sim_{a} Q$ by hypothesis, we have two possible ways that $Q$ may move. If $Q \stackrel{\beta}{\longmapsto} Q^{\prime}$ with $P^{\prime} \sim_{a} Q^{\prime}$, we reason as above. Otherwise $Q \stackrel{\tau}{\longmapsto} Q^{\prime}$ and $P^{\prime} \sim_{a} Q^{\prime} \mid \bar{b}\langle\underline{a}: \tilde{m} \| \tilde{c}\rangle^{\circ}$. Let then $Q \stackrel{\tau}{\longmapsto} Q^{\prime}$. By an inspection of the labelled transitions, there must exist $\hat{Q}$ and $\tilde{q}$ such that $Q \equiv(\boldsymbol{\nu} \tilde{q}) \hat{Q}$ and the move from $Q$ derives from two transitions $\hat{Q} \stackrel{\bar{\alpha}}{\longrightarrow} \cdot \stackrel{\alpha}{\longrightarrow}$ for suitable $\bar{\alpha}$ and $\alpha$. The proof proceeds with a case analysis on the format of $\alpha$ and $\bar{\alpha}$. Let then $\alpha=d(\underline{h}: \tilde{n} \| \tilde{e})^{\circ}$, and $\bar{\alpha}=\bar{d}\langle\underline{h}: \tilde{n} \| \tilde{e}\rangle^{\circ}$.

From $\hat{Q} \stackrel{\bar{\alpha}}{\longrightarrow}$ it follows that $\hat{Q} \stackrel{(i) \dagger \bar{\gamma}_{i}}{\longrightarrow}$ with $\bar{\gamma}_{i}=\bar{d}\langle\underline{h}: \tilde{g} \| \tilde{e}\rangle_{i}^{\circ}$, and $\tilde{g}=\tilde{n}$ or $\tilde{g}=\tilde{e}$ depending on the secrecy mode $\circ$. As a a consequence, $Q \equiv(\boldsymbol{\nu} \tilde{q}) \hat{Q} \stackrel{(\tilde{r}, i) \dagger \bar{\gamma}_{i}}{\longrightarrow} Q^{\prime \prime}$ with $\tilde{r}=\tilde{q} \cap\{d, \underline{h}, \tilde{g}, \tilde{e}\}$. From the hypothesis that $Q \sim_{a} P$, we then find a matching move $P \stackrel{(\tilde{r}, i) \dagger \bar{\gamma}_{i}}{\longrightarrow} P^{\prime \prime}$ with $P^{\prime \prime} \sim_{a} Q^{\prime \prime}$. Now we observe that the initial move $\beta$ from $P$ is still available on $P^{\prime \prime}$, i.e., $P^{\prime \prime} \stackrel{\beta}{\longmapsto}$. But then, we are back to the same situation as before, as this move from $P^{\prime \prime}$ must be matched by $Q^{\prime \prime}$ directly or via a silent action. This reasoning may be repeated only a finite number of times, after which $Q$ must be able to respond with a $\beta$ move: this is a consequence of our assumption that replication, and recursion, are guarded in our processes, hence $Q$ may not have infinitely many outputs ready to fire.

Without loss of generality, we assume that $Q$ responds with a $\beta$ move right after the first step, i.e. $Q^{\prime \prime} \stackrel{\beta}{\longmapsto}$ (in case the move occurs at a subsequent step, we simply repeat the argument used for the first step). Summarizing the reasoning above, we have:

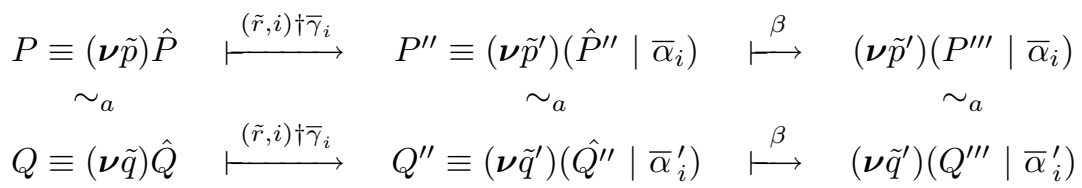

Here $\bar{\alpha}_{i}=\bar{d}\langle\underline{h}: \tilde{n} \| \tilde{e}\rangle_{i}^{\circ}$ is the indexed copy of $\bar{\alpha}$ indexed by $i$, and $\tilde{p}^{\prime}=\tilde{p} \backslash \tilde{r}, \tilde{q}^{\prime}=\tilde{q} \backslash \tilde{r}$. Similarly, $\bar{\alpha}_{i}^{\prime}=\bar{d}\left\langle\underline{h}: \tilde{n}^{\prime} \| \tilde{e}\right\rangle_{i}^{\circ}$ is the cached copy of the output emitted by $Q$ (notice that $\tilde{n}$ may be different from $\tilde{n}^{\prime}$ when $\circ$ is $\bullet$, even though the bitstring $\tilde{e}$ is the same as in $P$ ). Now, since $\beta$ is an input, from $P \stackrel{\beta}{\longmapsto}$ and $P \stackrel{(\tilde{r}, i) \dagger \bar{\gamma}_{i}}{\longrightarrow}$, it follows that $\{\tilde{r}, i\} \cap n(\beta)=\emptyset$. Hence from $Q \stackrel{(\tilde{r}, i) \dagger \bar{\gamma}_{i}}{\longmapsto} \cdot \stackrel{\beta}{\longmapsto}$ it also follows that $Q \stackrel{\beta}{\longmapsto} \cdot \stackrel{(\tilde{r}, i) \dagger \bar{\gamma}_{i}}{\longmapsto}$, and the same can be said of $P$. Thus, we have $P \stackrel{\beta}{\longmapsto} \equiv(\boldsymbol{\nu} \tilde{p})\left(P^{\prime \prime \prime} \mid \bar{\alpha}\right)$, and $Q \stackrel{\beta}{\longmapsto} \equiv(\boldsymbol{\nu} \tilde{q})\left(Q^{\prime \prime \prime} \mid \bar{\alpha}^{\prime}\right)$ where $\bar{\alpha}^{\prime}=\bar{d}\left\langle\underline{h}: \tilde{n}^{\prime} \| \tilde{e}\right\rangle^{\circ}$ is the output from $Q$ corresponding to $\bar{\alpha}$. To conclude, we must show that $(\boldsymbol{\nu} \tilde{p})\left(P^{\prime \prime \prime} \mid \bar{\alpha}\right) \sim_{a}(\boldsymbol{\nu} \tilde{q})\left(Q^{\prime \prime \prime} \mid \bar{\alpha}^{\prime}\right)$. If $\alpha$ is an authentic label (i.e. $\left.\underline{h}=h\right)$, by 
Lemma 4.2(2), we can complete the diagram above as follows:

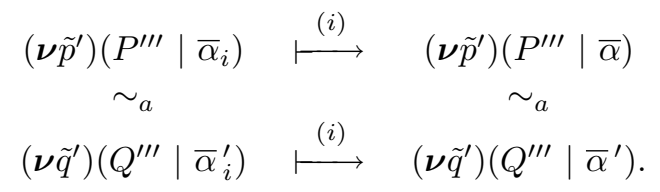

Here the desired relation follows because $\sim_{a}$ is closed by restriction, by Lemma 5.2. If instead $\alpha$ is non authentic $(h=-)$, again by Lemma $4.2(2)$, the diagram can continue as follows:

$$
\begin{array}{ccc}
\left(\boldsymbol{\nu} \tilde{p}^{\prime}\right)\left(P^{\prime \prime \prime} \mid \bar{\alpha}_{i}\right) & \stackrel{(i)}{\longmapsto} & \left(\boldsymbol{\nu} \tilde{p}^{\prime}\right)\left(P^{\prime \prime \prime}|\bar{\alpha}| \bar{\alpha}_{i}\right) \\
\sim_{a} & \sim_{a} \\
\left(\boldsymbol{\nu} \tilde{q}^{\prime}\right)\left(Q^{\prime \prime \prime} \mid \bar{\alpha}_{i}^{\prime}\right) & \stackrel{(i)}{\longmapsto} & \left(\boldsymbol{\nu} \tilde{q}^{\prime}\right)\left(Q^{\prime \prime \prime}\left|\bar{\alpha}^{\prime}\right| \bar{\alpha}_{i}^{\prime}\right)
\end{array}
$$

Then, by Lemma 5.1, we know that $\left(\boldsymbol{\nu} \tilde{p}^{\prime}\right)\left(P^{\prime \prime \prime} \mid \bar{\alpha}\right) \sim_{a}\left(\boldsymbol{\nu} \tilde{q}^{\prime}\right)\left(Q^{\prime \prime \prime} \mid \bar{\alpha}^{\prime}\right)$, and the claim follows again by closure under restriction (on the tuple $\tilde{r}$ ).

The syntactic restrictions imposed on our processes, in particular the absence of unguarded recursion and, similarly, of an unguarded choice operator are crucial in the proof of Theorem 5.1. Indeed, if we lift those restrictions, not only the proof breaks, but the theorem itself is false. We conclude the section with two counter-examples that substantiate this observation. Let $* R$ denote the replicated version of $R$, defined by the unguarded recursive equation $* R \stackrel{\text { def }}{=} * R \mid R$.

The first example shows that Theorem 5.1 is false in the presence of unguarded replication. Consider the two processes below:

$$
\begin{array}{lll}
P & \stackrel{\text { def }}{=} & Q \mid b(-: x) \cdot \bar{b}\langle-: x\rangle \\
Q & \stackrel{\text { def }}{=} & * \bar{a}\langle-: m\rangle \mid * a(-: x) \cdot \bar{a}\langle-: x\rangle
\end{array}
$$

Clearly $P \nsim Q$, because there is no way for $Q$ to match the input transition available for $P$ on $b$. On the other hand, the two processes cannot be distinguished in the asynchronous version of bisimilarity as $P$ 's move on $b, P \stackrel{b(-: n)}{\longrightarrow} Q \mid \bar{b}\langle-: n\rangle$, may be matched by $Q$ via a $\tau$-transition that takes $Q$ back to itself (thanks to the presence of the replicated output).

Formally, consider the following relation:

$$
\mathscr{R}=\{(\hat{Q} \mid b(-: x \| y) \cdot \bar{b}\langle-: x \| y\rangle, \hat{Q}) \mid \hat{Q} \text { is a derivative of } Q\} \cup I d
$$

Notice that $\hat{Q} \equiv Q \mid R$, where $R$ is the parallel composition of possibly replicated outputs on $a$ (both $\bar{a}\langle-: m\rangle$ and other outputs on $a$ read from the environment and resent by $Q$ ), with their relative indexed copies: this can be easily proved by induction on the length of the derivation from $Q$ to $\hat{Q}$. Thus $\hat{Q}$ cannot synchronize on $b$ and, since $Q \stackrel{\tau}{\longmapsto} Q$, we also have $\hat{Q} \stackrel{\tau}{\longmapsto} \hat{Q}$.

It is now trivial to verify that $\mathscr{R}$ is an asynchronous bisimulation. The only interesting case is $\hat{Q}|b(-: x \| y) . \bar{b}\langle-: x \| y\rangle \stackrel{b(-: n \| c)}{\longmapsto} \hat{Q}| \bar{b}\langle-: n \| c\rangle$ which is simulated by 
$\hat{Q} \stackrel{\tau}{\longmapsto} \hat{Q}$ with $\hat{Q}|\bar{b}\langle-: n \| c\rangle \mathscr{R} \hat{Q}| \bar{b}\langle-: n \| c\rangle$, since $I d \subseteq \mathscr{R}$. Given that $Q$ is a (zero) derivative of itself, we obtain that $P \mathscr{R} Q$ and thus $P \sim_{a} Q$.

A similar example shows that Theorem 5.1 fails if we extend the syntax with an unguarded nondeterministic choice operator, $P_{1}+P_{2}$, defined with the usual semantics $\left(P_{1}+P_{2} \stackrel{\alpha}{\longrightarrow} \eta P^{\prime}\right.$ if $P_{1} \stackrel{\alpha}{\longrightarrow} \eta P^{\prime}$ or $\left.P_{2} \stackrel{\alpha}{\longrightarrow} \eta P^{\prime}\right)$. Let $a(-: x) *$ denote the guarded recursive process $Q \stackrel{\text { def }}{=} a(-: x) . Q$, and consider the following processes:

$$
\begin{array}{lll}
P & \stackrel{\text { def }}{=} & a(-: x) * \mid(\bar{a}\langle-: x\rangle+b(-: x) \cdot \bar{b}\langle-: x\rangle) \\
Q & \stackrel{\text { def }}{=} & a(-: x) * \mid \bar{a}\langle-: x\rangle
\end{array}
$$

Clearly, we have $P \nsim Q$, because there is no way for $Q$ to match the input transition available for $P$ on $b$. On the other hand, the two processes cannot be distinguished in the asynchronous version of bisimilarity as $P \stackrel{b(-: n)}{\longrightarrow} a(-: x) * \mid \bar{b}\langle-: x\rangle$, may be matched by $Q \stackrel{\tau}{\longmapsto} a(-: x) *$.

\subsection{Characterizing Barbed Equivalence}

We conclude the section on bisimilarity showing that bisimilarity coincides with (our version of) barbed equivalence. For the soundness direction of the proof, we need a standard lemma connecting barbs with labelled transitions.

Lemma 5.3. $M \downarrow b$ if and only if $M \stackrel{(\tilde{n}, i) \dagger \bar{b}\langle\ldots\rangle_{i}^{\circ}}{\longrightarrow}$ with $b \notin \tilde{n}$.

Proof. In both directions, by an inspection of labelled transition system.

Theorem 5.2. For any pair of trusted processes, $P \sim Q$ implies $P \simeq Q$.

Proof. Define the candidate relation

$$
\mathscr{R}=\{((\boldsymbol{\nu} \tilde{n})(I \mid P),(\boldsymbol{\nu} \tilde{n})(I \mid Q)) \mid P \sim Q \text { with } P, Q \text { trusted derivatives, } I \text { intruder }\}
$$

We show that $\mathscr{R} \subseteq \simeq$. Being $I$ arbitrary, $\mathscr{R}$ is contextual by definition. That $\mathscr{R}$ is barb preserving follows easily by Lemma 5.3 above. In particular, from $(\boldsymbol{\nu} \tilde{n})(I \mid P) \downarrow b$ we know that $I \equiv(\boldsymbol{\nu} \tilde{m})\left(\bar{b}\langle\ldots\rangle^{\circ} \mid I^{\prime}\right)$ or $P \equiv(\boldsymbol{\nu} \tilde{m})\left(\bar{b}\langle\ldots\rangle^{\circ} \mid P^{\prime}\right)$, with $b \notin \tilde{m}, \tilde{n}$. In the first case, we have immediately $(\boldsymbol{\nu} \tilde{n})(I \mid Q) \downarrow b$. In the second case, $P \stackrel{(\tilde{m}, i) \bar{b}\langle\ldots\rangle_{i}^{\circ}}{\longmapsto}$ and, by the hypothesis $P \sim Q$, we have $Q \stackrel{(\tilde{m}, i) \bar{b}(\ldots)_{i}^{\circ}}{\longmapsto}$. Hence $Q \downarrow b$ and given that $b \notin \tilde{n}$, $(\boldsymbol{\nu} \tilde{n})(I \mid Q) \downarrow b$ as desired.

It remains to show that $\mathscr{R}$ is reduction closed. By Lemma 4.1, we may reason equivalently in terms of $\tau$-transitions (as opposed to reductions). Assume $(\boldsymbol{\nu} \tilde{n})(I \mid P) \stackrel{\tau}{\longmapsto} R$ : we must find a matching transition $(\boldsymbol{\nu} \tilde{n})(I \mid Q) \stackrel{\tau}{\longmapsto} R^{\prime}$ with $R \mathscr{R} R^{\prime}$. The proof is by cases on the derivation of the move from $(\boldsymbol{\nu} \tilde{n})(I \mid P)$.

If the move comes from $I$, then the same move is available from $(\boldsymbol{\nu} \tilde{n})(I \mid Q)$ and we are done. The case when the transition comes from $P \stackrel{\tau}{\longmapsto} P^{\prime}$ is equally simple: we just have to appeal to the hypothesis $P \sim Q$. 
The remaining cases are when both $I$ and $P$ contribute to the move. There are a multitude of cases, all with the same structure: we give the (Synch Intercept) cases as representatives. Assume then, that $(\boldsymbol{\nu} \tilde{n})(I \mid P) \stackrel{\tau}{\longmapsto} R$ because $P \stackrel{(\tilde{p}, i) \bar{b}\langle\underline{a}: \tilde{m} \| \tilde{c}\rangle_{i}^{\circ}}{\longmapsto} \hat{P}$, $I \stackrel{\dagger b(\underline{a}: \tilde{m} \| \tilde{c})^{\circ}}{\longrightarrow} \hat{I}$ and $R$ is $(\boldsymbol{\nu} \tilde{n}, \tilde{p}, i)(\hat{I} \mid \hat{P})$. From the hypothesis $P \sim Q$, we know that $Q \stackrel{(\tilde{p}, i) \bar{b}\langle\underline{a}: \tilde{m} \| \tilde{c}\rangle_{i}^{\circ}}{\longmapsto} \hat{Q}$, with $\hat{P} \sim \hat{Q}$. We are done as $(\boldsymbol{\nu} \tilde{n})(Q \mid I) \stackrel{\tau}{\longmapsto}(\boldsymbol{\nu} \tilde{n}, \tilde{p}, i)(\hat{I} \mid \hat{Q})$ and $(\boldsymbol{\nu} \tilde{n}, \tilde{p}, i)(\hat{I} \mid \hat{Q})$ is the desired $R^{\prime}$.

We continue with the completeness part of the characterization proof, showing that bisimilarity is implied by barbed equivalence. As usual, the proof amounts to showing that the actions involved in the labelled transitions of a process are definable by corresponding, testing contexts that provoke those actions. The following, auxiliary lemma allows us to 'strip away' the residuals of such testing contexts.

Lemma 5.4. Let $P, Q$ be trusted processes, $\tilde{n} \subseteq \mathbf{N}_{t}$ and $e \in \mathbf{N}_{u}$ fresh in $P, Q$. Then $(\boldsymbol{\nu} \tilde{n})(P \mid \bar{e}\langle-: \tilde{n} \| \tilde{n}\rangle) \simeq(\boldsymbol{\nu} \tilde{n})(Q \mid \bar{e}\langle-: \tilde{n} \| \tilde{n}\rangle)$ implies $P \simeq Q$.

Proof. By coinduction, define:

$$
\begin{aligned}
\mathscr{R}=\{(M, N) \mid & \text { there exist } \tilde{n} \subseteq \mathbf{N}_{t}, e \in \mathbf{N}_{u} \text { fresh in } M, N \text { such that } \\
& (\boldsymbol{\nu} \tilde{n})(M \mid \bar{e}\langle-: \tilde{n} \| \tilde{n}\rangle) \simeq(\boldsymbol{\nu} \tilde{n})(N \mid \bar{e}\langle-: \tilde{n} \| \tilde{n}\rangle)\}
\end{aligned}
$$

Clearly $\mathscr{R}$ is symmetric. We show that it is an intruder bisimulation.

- $\mathscr{R}$ is barb preserving. Assume $M \downarrow b$. The interesting case is when $b \in \tilde{n}$. Let then $b$ be the $j$-th element in the tuple $\tilde{n}, f \neq e$ a name fresh in $M$ and $N$, and define ${ }^{\dagger}$ :

$$
I \stackrel{\text { def }}{=} e(-: \tilde{x} \| \tilde{y}) \cdot \dagger z(\ldots)_{i}^{\circ} \text {.if } z=x_{j} \text { then } \bar{f}\langle-: \|\rangle \text { else } \mathbf{0}
$$

Then $(\boldsymbol{\nu} \tilde{n})(M \mid \bar{e}\langle-: \tilde{n} \| \tilde{n}\rangle) \mid I \longrightarrow M_{0} \longrightarrow M_{1}$ with $M_{0} \not \downarrow f$, and $M_{1} \downarrow f$. Now, from the hypothesis $(\boldsymbol{\nu} \tilde{n})(M \mid \bar{e}\langle-: \tilde{n} \| \tilde{n}\rangle) \simeq(\boldsymbol{\nu} \tilde{n})(N \mid \bar{e}\langle-: \tilde{n} \| \tilde{n}\rangle)$ we find $N_{0}$ and $N_{1}$ such that that $(\boldsymbol{\nu} \tilde{n})(N|\bar{e}\langle-: \tilde{n} \| \tilde{n}\rangle| I) \longrightarrow N_{0} \longrightarrow N_{1}$ and $N_{0} \not \subset f, N_{1} \downarrow f$. This, in turn, implies $N \downarrow b$ as desired.

- $\mathscr{R}$ is reduction closed. Assume $M \longrightarrow M^{\prime}$. Then we have a corresponding transition $(\boldsymbol{\nu} \tilde{n})(M \mid \bar{e}\langle-: \tilde{n} \| \tilde{n}\rangle) \longrightarrow(\boldsymbol{\nu} \tilde{n})\left(M^{\prime} \mid \bar{e}\langle-: \tilde{n} \| \tilde{n}\rangle\right)$, and from the hypothe$\operatorname{sis}(\boldsymbol{\nu} \tilde{n})(M \mid \bar{e}\langle-: \tilde{n} \| \tilde{n}\rangle) \simeq(\boldsymbol{\nu} \tilde{n})(N \mid \bar{e}\langle-: \tilde{n} \| \tilde{n}\rangle)$ there must exist $\hat{N}$ such that $(\boldsymbol{\nu} \tilde{n})(N \mid \bar{e}\langle-: \tilde{n} \| \tilde{n}\rangle) \longrightarrow \hat{N}$ and $\hat{N} \simeq(\boldsymbol{\nu} \tilde{n})\left(M^{\prime} \mid \bar{e}\langle-: \tilde{n} \| \tilde{n}\rangle\right)$ Thus $\hat{N} \downarrow e$ and since $e$ is fresh in $N, M$, this implies $\hat{N} \equiv(\boldsymbol{\nu} \tilde{n})\left(N^{\prime} \mid \bar{e}\langle-: \tilde{n} \| \tilde{n}\rangle\right)$ with $N \longrightarrow N^{\prime}$, as desired.

- $\mathscr{R}$ is contextual. Assume $(M, N) \in \mathscr{R}$.

We first show that $(M|I, N| I) \in \mathscr{R}$ for all intruder contexts $I$. Since $\simeq$ is contextual, we know that $(\boldsymbol{\nu} \tilde{n})(M \mid \bar{e}\langle-: \tilde{n} \| \tilde{n}\rangle)\left|I^{\prime} \simeq(\boldsymbol{\nu} \tilde{n})(N \mid \bar{e}\langle-: \tilde{n} \| \tilde{n}\rangle)\right| I^{\prime}$, for all $I^{\prime}$. Now choose $e^{\prime} \in \mathbf{N}_{u}$ fresh in $M, N, I$ and let

$$
I^{\prime}=e(-: \tilde{x} \| \tilde{y}) \cdot\left(I[\tilde{x} / \tilde{n}] \mid \bar{e}^{\prime}\langle-: \tilde{x} \| \tilde{x}\rangle\right)
$$

\footnotetext{
$\dagger$ We are loose here as we do not specify the arity and the mode of the intercept prefix. Indeed, $M \downarrow b$ can derive from a plain or secret output of an arbitrary number of messages on $b$; for the argument to go through, after inputing on $a, I$ should run as many copies of process guarded by the input prefix as there are arities in $P$ and $Q$, for the two possible modes of the intercept prefix.
} 
Since $I$ is adversarial, and $\tilde{n} \subseteq \mathbf{N}_{t} I[\tilde{x} / \tilde{n}]$ is still a legal intruder process term. Now $(\boldsymbol{\nu} \tilde{n})(M \mid \bar{e}\langle-: \tilde{n} \| \tilde{n}\rangle) \mid I^{\prime} \longrightarrow(\boldsymbol{\nu} \tilde{n})\left(M|I| \bar{e}^{\prime}\langle-: \tilde{n} \| \tilde{n}\rangle\right)$. From the hypothesis $(\boldsymbol{\nu} \tilde{n})(M \mid \bar{e}\langle-: \tilde{n} \| \tilde{n}\rangle) \simeq(\boldsymbol{\nu} \tilde{n})(N \mid \bar{e}\langle-: \tilde{n} \| \tilde{n}\rangle)$ and the fact that $e^{\prime}$ is fresh, we find a corresponding transition $(\boldsymbol{\nu} \tilde{n})(N \mid \bar{e}\langle-: \tilde{n} \| \tilde{n}\rangle) \mid I^{\prime} \longrightarrow(\boldsymbol{\nu} \tilde{n})\left(N|I| \bar{e}^{\prime}\langle-: \tilde{n} \| \tilde{n}\rangle\right)$, with $(\boldsymbol{\nu} \tilde{n})\left(M|I| \bar{e}^{\prime}\langle-: \tilde{n} \| \tilde{n}\rangle\right) \simeq(\boldsymbol{\nu} \tilde{n})\left(N|I| \bar{e}^{\prime}\langle-: \tilde{n} \| \tilde{n}\rangle\right)$. Thus $(M|I, N| I) \in \mathscr{R}$ as desired.

It remains to show that $((\boldsymbol{\nu} m) M,(\boldsymbol{\nu} m) N) \in \mathscr{R}$ for all $m$. Given any such $m$, choose an untrusted $e^{\prime} \neq m$ fresh in $M, N$. Given that $e$ is also fresh in $M, N$, from the hypothesis $(\boldsymbol{\nu} \tilde{n})(M \mid \bar{e}\langle-: \tilde{n} \| \tilde{n}\rangle) \simeq(\boldsymbol{\nu} \tilde{n})(N \mid \bar{e}\langle-: \tilde{n} \| \tilde{n}\rangle)$ we know that $(\boldsymbol{\nu} \tilde{n})\left(M \mid \bar{e}^{\prime}\langle-: \tilde{n} \| \tilde{n}\rangle\right) \simeq$ $(\boldsymbol{\nu} \tilde{n})\left(N \mid \bar{e}^{\prime}\langle-: \tilde{n} \| \tilde{n}\rangle\right)$. Now we have: $(\boldsymbol{\nu} \tilde{n})\left((\boldsymbol{\nu} m) M \mid \bar{e}^{\prime}\langle-: \tilde{n} \| \tilde{n}\rangle\right) \equiv(\boldsymbol{\nu} m, \tilde{n})\left(M \mid \bar{e}^{\prime}\langle-\right.$ : $\tilde{n} \| \tilde{n}\rangle) \simeq(\boldsymbol{\nu} m, \tilde{n})\left(N \mid \bar{e}^{\prime}\langle-: \tilde{n} \| \tilde{n}\rangle\right) \equiv(\boldsymbol{\nu} \tilde{n})\left((\boldsymbol{\nu} m) N \mid \bar{e}^{\prime}\langle-: \tilde{n} \| \tilde{n}\rangle\right)$. This implies $((\boldsymbol{\nu} m) M,(\boldsymbol{\nu} m) N) \in \mathscr{R}$ as desired.

Theorem 5.3. For any pair of trusted processes, $P \simeq Q$ implies $P \sim Q$.

Proof. We show that $P \simeq Q$ implies $P \sim_{a} Q$, and conclude by Theorem 5.1. Let $\mathscr{R}=$ $\{(P, Q) \mid P \simeq Q\}$. We prove that $\mathscr{R}$ is an asynchronous bisimulation. Take $(P, Q) \in \mathscr{R}$ and let $P \stackrel{\alpha}{\longmapsto} P^{\prime}$. We must find a matching transition for $Q$.

If $\alpha=\tau$ the claim follows by Lemma 4.1 and the fact that $\simeq$ is reduction closed. If $\alpha$ is an input action, an inspection of the observable transitions shows that $\alpha=b(a: \tilde{m} \| \tilde{c})^{\circ}$ with $a \in \mathbf{N}_{u}$. Thus $\bar{\alpha}$ is a legal intruder context, and hence by contextuality $P|\bar{\alpha} \simeq Q| \bar{\alpha}$. Now, by construction, $P \mid \bar{\alpha} \longrightarrow P^{\prime}$, and by reduction closure we know that $Q \mid \bar{\alpha} \longrightarrow N$ with $N \simeq P^{\prime}$. We have the two expected possible cases: indeed, either $N \equiv Q^{\prime}$ with $Q \stackrel{\alpha}{\longmapsto} Q^{\prime}$, or $Q \stackrel{\tau}{\longmapsto} Q^{\prime}$ and $N \equiv Q^{\prime} \mid \bar{\alpha}$.

When $\alpha$ is an output or intercept action the proof proceeds by exhibiting a distinguishing context for each possible transition. We prove the intercept case as a representative. Also, to simplify the notation we restrict the proof to the simpler case when the intercepted payload (correspondingly the bitstring) is a monadic message. The extension to the polyadic case presents no difficulty, though it is notationally costly. Let then $\alpha=(\tilde{n}, i) \dagger \overline{b_{0}}\left\langle b_{1}: b_{2} \| b_{3}\right\rangle_{i}$, and let $\tilde{m}=f n(P, Q)$. Define:

$$
\begin{aligned}
I_{\tilde{m}}= & \overline{\mathrm{ko}}\langle-: \|\rangle \mid \\
& \dagger x_{0}\left(x_{1}: x_{2} \| x_{3}\right)_{i} \text {.if } \operatorname{MATCH}(\tilde{x}, \tilde{b}, \tilde{m}, \tilde{n}) \text { then } \operatorname{ko}(-: \|) . \overline{\mathrm{ok}}\langle-: \tilde{n} \| \tilde{n}\rangle \text { else } \mathbf{0}
\end{aligned}
$$

where ko and ok are chosen fresh, and MATCH is the composite conditions that identifies the label alpha univocally. In particular, $\operatorname{MATCH}(\tilde{x}, \tilde{b}, \tilde{b}, \tilde{n})$ corresponds to the following test (we omit the details of how such test can be encoded in terms of cascaded conditionals):

$$
\left(x_{j}=b_{j}\right)_{b_{j} \notin \tilde{n}} \wedge\left(x_{j} \notin \tilde{m}\right)_{b_{j} \in \tilde{n}} \wedge\left(x_{j}=x_{k}\right)_{b_{j}=b_{k} \in \tilde{n}} \wedge\left(x_{j} \neq x_{k}\right)_{b_{j} \neq b_{k} \in \tilde{n}}
$$

By construction, we have:

$$
P \mid I_{\tilde{m}} \longrightarrow M_{0} \longrightarrow M_{1} \equiv(\boldsymbol{\nu} \tilde{n})\left(P^{\prime} \mid \overline{\mathrm{ok}}\langle-: \tilde{n} \| \tilde{n}\rangle\right)
$$

Here, as a result of the interception, the opponent caches a copy of the message inter- 
cepted, namely $\overline{b_{0}}\left\langle b_{1}: b_{2} \| b_{2}\right\rangle_{i}^{\bullet}$. In the labelled transition, this copy is attributed to the derivative of $P$, that is $P^{\prime}$, explaining the structure of $M_{1}$.

Now observe that $M_{0} \downarrow$ ko, $M_{0} \not$ ok, and dually $M_{1} \not$ ko, $M_{1} \downarrow$ ok. Then, from the hypothesis $P \simeq Q$, we derive $P\left|I_{\tilde{m}} \simeq Q\right| I_{\tilde{m}}$. Hence there exist $N_{0}, N_{1}$ such that $Q \mid I_{\tilde{m}} \longrightarrow N_{0} \longrightarrow N_{1}$ and $M_{i} \simeq N_{i}(i=0,1)$. This, in turn, implies that $I_{\tilde{m}}$ must have consumed the input on ko. Thus, given that ko is fresh to $Q$, we know that there exists $Q^{\prime}$ such that $N_{1} \equiv(\boldsymbol{\nu} \tilde{n})\left(Q^{\prime} \mid \overline{\mathrm{ok}}\langle-: \tilde{n} \| \tilde{n}\rangle\right)$ and $Q \stackrel{\alpha}{\longmapsto} Q^{\prime}$. This is the matching transition we were looking for as, by by Lemma 5.4 we know that $M_{1} \simeq N_{1}$ implies $P^{\prime} \simeq Q^{\prime}$, as desired.

As usual, the characterization of barbed equivalence in terms of bisimilarity represents a useful result, as it allows us to carry out coinductive proofs of barbed equivalence for processes. Indeed, bisimilarity turns our very effective as a proof technique for most of the standard security equivalences for secrecy and authentication. In addition, based on the results in Section 6.2, the coinductive proofs are fairly elegant, because based on rather "small" candidates. We will return on this briefly at the end of Section 6.2.

We conclude this section by exemplifying the use of the coinductive characterization of barbed equivalence in the proof of Theorem 3.1, that we state again below.

Theorem 5.4 (Congruence over trusted processes). Let $P, Q$ be trusted processes. $P \simeq Q$ implies $P|R \simeq Q| R$, for all trusted processes $R$ that do not impersonate any identity in $f n(P, Q)$.

Proof. We first need the following observation. Let $M$ and $N$ be two network processes, and let $\sigma$ be an injective substitution that maps any subset of the trusted free names in $M, N$ onto corresponding untrusted names: then $M \sigma \simeq N \sigma$ implies $M \simeq N$. We can show, equivalently, $M \sigma \sim N \sigma$ implies $M \sim N$ and this, in turn, follows directly by coinduction, noting that $M \stackrel{\alpha}{\longmapsto} M^{\prime}$ implies $M \sigma \stackrel{\alpha \sigma}{\longmapsto} M^{\prime} \sigma$ and conversely, that when $\alpha$ is observable, $M \sigma \stackrel{\alpha \sigma}{\longmapsto} M^{\prime} \sigma$ implies $M \stackrel{\alpha}{\longmapsto} M^{\prime}$ (clearly, the same is true of $N$ ).

Now choose $\sigma$ to be an injective substitution that maps all the trusted names impersonated by $R$ into corresponding untrusted names ( $\sigma$ is the identities on all the other names). By construction, $R \sigma$ is an intruder, and since $R$ does not impersonate any identity in $f n(P, Q)$, we have that $P \sigma=P$, and $Q \sigma=Q$. From the hypothesis $P \simeq Q$, by contextuality, it also follows $P|R \sigma \simeq Q| R \sigma$, hence $(P \mid R) \sigma \simeq(Q \mid R) \sigma$. By the previous observation, this implies $P|R \simeq Q| R$ as desired.

\section{More on intruders}

Before exploring in further detail the security applications of the calculus, and the import of our observational equivalence in specifying security goals, in this section we conduct an in-depth analysis of our intruder model, and contrast it with other models found in the security literature. Specifically, we analyze two further models that arise from endowing the intruders with (i) different adversarial capabilities and (ii) increasingly powerful control on the interaction among the distributed principals of a network. As a result of this analysis we will also derive powerful proof techniques for bisimilarity. 


\subsection{Eavesdroppers}

Standard formalizations of Dolev-Yao models assume that the intruder has the ability to "tap the wire" and silently eavesdrop on network traffic without necessarily intercepting it. We extend the set of adversarial forms to provide a formal account of this stronger model of intruder, and analyze the expressiveness of this primitive in terms of the discriminating power it conveys.

The syntax of the high-level calculus is unchanged from Section 2, while the new productions for networks are as follows:

$$
M, N::=\ldots(\text { as in Section } 2) \ldots \mid ? z(x: \tilde{y} \| \tilde{w})_{i}^{\circ} \cdot M
$$

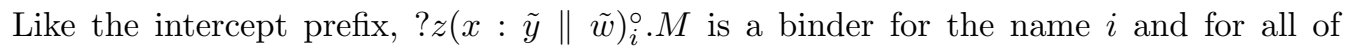
its component variables, with scope $M$. The reductions and labelled transitions follow the exact same rationale as the corresponding rules for the intercept primitive, with the differences (i) that eavesdropping does not consume the output, and hence (ii) that it does not create a copy in case the output is authentic (cf. Appendix B).

In the rest of this section we analyze the import of eavesdropping on the notion of bisimilarity that results from its inclusion in the calculus. In that direction we let $\left(\sim^{\kappa}\right)_{\kappa \subseteq\{\dagger, ?, !\}}$ denote the family of bisimilarity relationships associated with the corresponding set of adversarial primitives. Similarly, we define the set $\left(\sim_{a}^{\kappa}\right)_{\kappa \subseteq\{\dagger, ?, !\}}$ for the asynchronous setting, and look at the relative strength of (some of) the equivalences in these sets.

We first that eavesdropping does not give any additional discriminatory power.

Theorem 6.1. $\sim_{a}^{\dagger !}=\sim_{a}^{? \dagger !}$, and similarly $\sim^{\dagger !}=\sim^{? \dagger !}$.

Proof. We outline the proof the the synchronous relation. The reasoning for the asynchronous case is similar.

That $\sim^{? \dagger !} \subseteq \sim^{\dagger !}$ is obvious, as $\left\{(P, Q) \mid P \sim^{? \dagger !} Q\right\}$ is trivially a $\dagger$ !-bisimulation. For the reverse inclusion, we use the candidate $\mathscr{R}=\left\{(P, Q) \mid P \sim^{\dagger !} Q\right\}$ and show that it is a ?†!-bisimulation.

Take $(P, Q) \in \mathscr{R}$ and let $P \stackrel{\alpha}{\longmapsto} P^{\prime}$. The only interesting cases are when $\alpha$ is an eavesdrop action: we show the case when $\alpha=(\tilde{r}, i) ? \bar{b}\langle-: \tilde{c} \| \tilde{c}\rangle_{i}^{\bullet}$. as representative. In this case we have $P \equiv(\boldsymbol{\nu} \tilde{p}, \tilde{r})\left(\hat{P} \mid \bar{b}\langle-: \tilde{m} \| \tilde{c}\rangle^{\bullet}\right)$ and $P^{\prime} \equiv(\boldsymbol{\nu} \tilde{p})\left(\hat{P}|\bar{b}\langle-: \tilde{m} \| \tilde{c}\rangle \bullet| \bar{b}\langle-: \tilde{m} \| \tilde{c}\rangle_{i}^{\bullet}\right)$ for a suitable tuple $\tilde{m}$. Then we may reason as follows to find a matching transition from $Q$ :

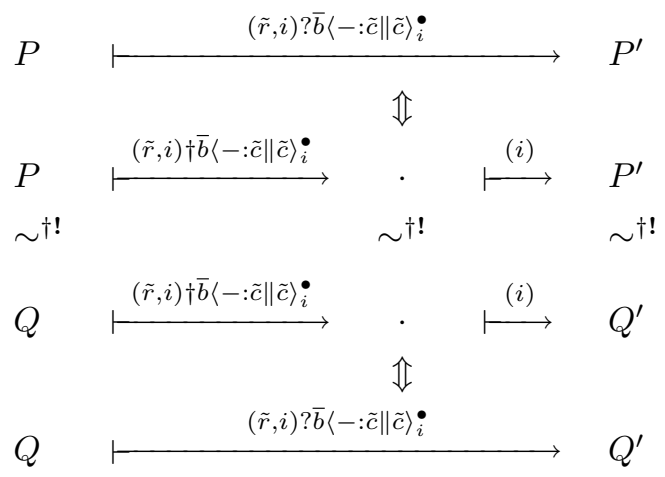


Notice that we rely on a one-to-one correspondence between eavesdrop actions and sequences of intercept-replay (or forward, in case of authenticated outputs). That an eavesdrop may be simulated by an intercept-replay (forward) sequence follows by an inspection of the labelled transition. For the opposite direction, we further need an appeal to Lemma 4.2(2), to get a guarantee that the reply (forward) selects the unique indexed output stored by intercept.

Next, we show that eavesdropping is strictly less powerful than intercepting.

Theorem 6.2. $\sim_{a}^{\dagger !} \subsetneq \sim_{a}^{? !}$ and similarly $\sim^{\dagger !} \subsetneq \sim^{? !}$.

Proof. Clearly $\sim_{a}^{?+!} \subseteq \sim_{a}^{? !}$, and this implies $\sim_{a}^{\dagger !} \subseteq \sim_{a}^{? !}$ since, by Theorem 6.1, we have $\sim_{a}^{?+!}=\sim_{a}^{\dagger !}$. The exact same reasoning applies in the synchronous case. That the inclusions are strict follows by the following counter-example, which applies uniformly to the synchronous and asynchronous cases. Let $a \in \mathbf{N}_{t}$, and take the following two processes:

$$
\begin{array}{lll}
P & \stackrel{\text { def }}{=} & \bar{a}\langle-: m\rangle|\bar{a}\langle-: m\rangle| * a(-: x) \cdot \bar{a}\langle-: x\rangle \\
Q & \stackrel{\text { def }}{=} & \bar{a}\langle-: m\rangle \mid * a(-: x) \cdot \bar{a}\langle-: x\rangle
\end{array}
$$

$P \chi_{a}^{? ! !} Q$, because $P$ and $Q$ may be distinguished using the intercept moves to count the outputs in the two processes. On the other hand, $P \sim^{? !} Q$ as counting in not possible with eavesdrop moves, because eavesdropping does not consume the output. The only remaining possibility to tell $P$ from $Q$ would be to consume the outputs by output moves, but this is not possible because $a$ is a trusted name, hence there are no observable output moves on $a$. In fact, the intruder cannot input on a trusted name $a$ but only eavesdrop or intercept the communication.

\section{2. $M$ en in the middle}

We continue our analysis by looking at man-in-the-middle intruder adopted in (Adão and Fournet, 2006). In this new model, two principals may never engage in a synchronization directly like in our initial semantics of Section 3. Instead, all exchanges require the mediation of the intruder which intercepts all outputs and then delivers them to the processes in the exact moment they are ready to consume them.

A man-in-the-middle intruder is easily accounted for in our calculus. The reduction relation arises from the relation defined in Table 1 by dropping the (Comm) rule and by replacing the (Forward) and (Replay) rules with two rules in Table 4. A corresponding modification is required on the labelled transition semantics to mimic the form of threeway synchronization induced by the new rules of reduction. In particular, the new labelled transitions arise from those defined in Table 3 by $(i)$ replacing the rules (Co-reply) and (Co-forward) with the two rules in Table 4, and (ii) by dropping the (Synch) rule (thus effectively disabling direct synchronization between trusted processes). The observable LTS for the new semantics is derived exactly as we did in Definition 5.1.

In the rest of this section we study the relative strength of the notions of bisimilarity resulting in the intruder models, based on their labelled transition semantics, and the 
Table 4 Man-in-the-middle semantics

\section{Reductions:}

(Forward) $\quad \bar{b}\langle a: \tilde{m} \| \tilde{c}\rangle_{i}^{\circ}\left|!_{i}\right| b(a: \tilde{y} \| \tilde{z})^{\circ} . N \quad \longrightarrow \quad N\{\tilde{m} / \tilde{y}, \tilde{c} / \tilde{z}\}$

(Replay) $\quad \bar{b}\langle-: \tilde{m} \| \tilde{c}\rangle_{i}^{\circ}|! i| b(-: \tilde{y} \| \tilde{z})^{\circ} . N \quad \longrightarrow \quad \bar{b}\langle-: \tilde{m} \| \tilde{c}\rangle_{i}^{\circ} \mid N\{\tilde{m} / \tilde{y}, \tilde{c} / \tilde{z}\}$

\section{Labelled Transitions}

$$
\begin{array}{cc}
\text { (Co-replay) } & \text { (Co-forward) } \\
\bar{b}\langle-: \tilde{m} \| \tilde{c}\rangle_{i}^{\circ} \mid N \stackrel{b^{(i)} \stackrel{(-: \tilde{m} \| \tilde{c})^{\circ}}{\longrightarrow} N^{\prime}\langle-: \tilde{m} \| \tilde{c}\rangle_{i}^{\circ} \mid N^{\prime}}{ } & \bar{b}\langle a: \tilde{m} \| \tilde{c}\rangle_{i}^{\circ} \mid N \stackrel{(i)}{\longrightarrow} N^{\prime}
\end{array}
$$

associated notions of bisimilarity. To avoid ambiguity between the two labelled transition systems, and the resulting notions of bisimilarity we adopt the following notation. We note $\stackrel{\alpha}{\longrightarrow}$ DY (respectively $\stackrel{\alpha}{\longmapsto}$ DY ) the (observable) labelled transition relation for the Dolev-Yao model, resulting from the transitions in Table 3. On the other hand, we note with $\stackrel{\alpha}{\longrightarrow}$ мгм and $\stackrel{\alpha}{\longmapsto}$ мтм the relations for the Man-in-the-middle model, resulting from the LTS formed as described in $(i)$ and $(i i)$ above. Finally, we note $\stackrel{\text { DY }}{\sim}$ and $\stackrel{\text { MIM }}{\sim}$ the associated notions of (synchronous) bisimilarity (notice, to this regard, that having disabled all $\tau$ actions on trusted processes, the relations of asynchronous and synchronous bisimilarity on trusted processes collapse to the same relation $\stackrel{\text { MIM }}{\sim}$ ).

At a first look, the new equivalence $\stackrel{\text { MTM }}{\sim}$ would appear finer than $\stackrel{\text { DY }}{\sim}$ due to the tighter control the new intruder can exercise over the interaction between the principals of a network. As it turns out, however, this additional control does not add any discriminating power.

We start noting that the simple properties on the structure of the indexed copies occurring in a process, proved in Section 4, extend to the new labelled transitions. In particular Lemma 4.2 holds just as well when $P$ is a MIM-derivative of a trusted process, and Lemma 5.1 is true also of $\stackrel{\text { MIM }}{\sim}$. Next, we introduce a definition that identifies a useful binary relation on processes and their derivatives. Throughout the section we tacitly assume that all the processes we refer to (as well as their run-time derivatives) are trusted.

Definition 6.1 (Compatible processes). $P$ and $Q$ are index compatible, or simply compatible, if $P \equiv(\boldsymbol{\nu} \tilde{p})\left(\hat{P} \mid \bar{b}\langle\underline{a}: \tilde{m} \| \tilde{c}\rangle_{i}^{\circ}\right)$ if and only if $Q \equiv(\boldsymbol{\nu} \tilde{q})\left(\hat{Q} \mid \bar{b}\left\langle\underline{a}: \tilde{m}^{\prime} \| \tilde{c}\right\rangle_{i}^{\circ}\right)$, where $\tilde{m}=\tilde{m}^{\prime}$ whenever $\circ=\varepsilon$ or $b \in \mathbf{N}_{u}$.

By Lemma 4.2, each index of a trusted process indexes exactly one indexed message. Consequently, assuming two processes compatible implies that we may establish a bijection between their indexed messages: indeed the indexed messages in the two processes have the same structure, up-to their respective payload which may be different only when the messages are encrypted. As we shall see, compatibility is a convenient property that we will often presuppose of the processes included in the candidate relations used in 
the proofs of this section. Given that all static trusted processes are pairwise compatible (because they have no indexed message) the assumption will not involve any loss of generality for the results we will prove on static trusted processes.

The compatibility relation is closed under the transition relation, both in the Dolev-Yao and Man-in-the-middle transition systems, in the following sense.

Lemma 6.1. Let $P$ and $Q$ be compatible processes. If $P \stackrel{\alpha}{\longmapsto} \eta P^{\prime}$ and $Q \stackrel{\alpha}{\longmapsto} \eta Q^{\prime}$, then $P^{\prime}$ and $Q^{\prime}$ are compatible, with $\eta$ any of DY and MIM.

Proof. In Appendix A

A further lemma shows that bisimilarity (in either intruder model) is insensitive to the choice of the indexes associated with the intercepted outputs, and to the duplication with a different index of existing indexed copies of non-authentic messages.

Lemma 6.2. Let $\gamma_{i}=\bar{a}\langle\underline{b}: \tilde{m} \| \tilde{c}\rangle_{i}^{\circ}$ and $\gamma_{i}^{\prime}=\bar{a}\left\langle\underline{b}: \tilde{m}^{\prime} \| \tilde{c}\right\rangle_{i}^{\circ}$, and let $P_{i}$ and $Q_{i}$ be the two trusted derivatives $P_{i} \equiv(\boldsymbol{\nu} \tilde{p})\left(\hat{P} \mid \gamma_{i}\right)$ and $Q_{i} \equiv(\boldsymbol{\nu} \tilde{q})\left(\hat{Q} \mid \gamma_{i}^{\prime}\right)$. If $P_{i} \stackrel{\eta}{\sim} Q_{i}$ then, for any $j \notin f n\left(P_{i}, Q_{i}\right) \cup\{\tilde{p}, \tilde{q}\}$, we have:

$1 \quad P_{j} \equiv(\boldsymbol{\nu} \tilde{p})\left(\hat{P} \mid \gamma_{j}\right) \stackrel{\eta}{\sim}(\boldsymbol{\nu} \tilde{q})\left(\hat{Q} \mid \gamma_{j}^{\prime}\right) \equiv Q_{j}$

$2 \quad P_{i, j} \equiv(\boldsymbol{\nu} \tilde{p})\left(\hat{P}\left|\gamma_{i}\right| \gamma_{j}\right) \stackrel{\eta}{\sim}(\boldsymbol{\nu} \tilde{q})\left(\hat{Q}\left|\gamma_{i}^{\prime}\right| \gamma_{j}^{\prime}\right) \equiv Q_{i, j}$ when $\underline{b}=-$

where $\stackrel{\eta}{\sim}$ is either $\stackrel{\text { DY }}{\sim}$ or $\stackrel{\text { MIM }}{\sim}$, and $\gamma_{j}$ and $\gamma_{j}^{\prime}$ are $\gamma$ and $\gamma^{\prime}$ indexed by $j$ rather than $i$.

Proof. In Appendix A.

The next Lemma proves another useful closure property, this time for $\stackrel{\text { MM }}{\sim}$ under the (co-reply) and (co-forward) transitions in the DY-system.

Lemma 6.3. Assume $P$ and $Q$ compatible: if $P \stackrel{\text { MIM }}{\sim} Q$ and $P \stackrel{(i)}{\longmapsto}$ DY $P^{\prime}$ then $Q \stackrel{(i)}{\longmapsto}{ }_{\mathrm{DY}} Q^{\prime}$ and $P^{\prime} \stackrel{\text { MIM }}{\sim} Q^{\prime}$.

Proof. In Appendix A.

Theorem 6.3. On trusted processes $\stackrel{\text { MIM }}{\sim} \stackrel{\text { DP }}{\sim}$.

Proof. We show that the following relation is a (synchronous) DY-bisimulation:

$$
\mathscr{R}=\{(P, Q) \mid P \stackrel{\text { MIM }}{\sim} Q \text { with } P, Q \text { compatible }\} .
$$

Let $(P, Q) \in \mathscr{R}$ and $P \stackrel{\alpha}{\longmapsto}{ }_{\mathrm{DY}} P^{\prime}$ : we must find a matching transition $Q \stackrel{\alpha}{\longmapsto_{\mathrm{DY}}} Q^{\prime}$ with $P^{\prime} \stackrel{\text { Mim }}{\sim} Q^{\prime}$. The fact that $P^{\prime}$ and $Q^{\prime}$ are compatible directly derive from Lemma 6.1 . We reason by cases on the format of $\alpha$. When $\alpha \notin\{\tau,(i)\}$, we have $P \stackrel{\alpha}{\longmapsto_{\text {DY }}} P^{\prime}$ iff $P \stackrel{\alpha}{\longmapsto_{\text {MIM }}} P^{\prime}$. From $P \stackrel{\text { MIM }}{\sim} Q$, we know that $Q \stackrel{\alpha}{\longmapsto}$ MIM $Q^{\prime}$, with $P^{\prime} \stackrel{\text { MIM }}{\sim} Q^{\prime}$. We are done since $Q \stackrel{\alpha}{\longmapsto} \longmapsto_{\text {MIM }} Q^{\prime}$ iff $Q \stackrel{\alpha}{\longmapsto} Q_{\text {DY }}^{\prime}$.

When $\alpha=(i)$, the proof follows directly by Lemma 6.3. Assume then $\alpha=\tau$. The transition $P \stackrel{\tau}{\longmapsto}$ DY $P^{\prime}$ must be derived from two transitions of the form

$$
\hat{P} \stackrel{\bar{b}\langle\underline{a}: \tilde{m} \| \tilde{c}\rangle^{\circ}}{\longrightarrow} \mathrm{DY} \cdot \stackrel{b(\underline{a}: \tilde{m} \| \tilde{c})^{\circ}}{\longrightarrow} \hat{P}^{\prime}
$$


where $P \equiv(\boldsymbol{\nu} \tilde{p}) \hat{P}$ and $P^{\prime} \equiv(\boldsymbol{\nu} \tilde{p}) \hat{P}^{\prime}$. We distinguish two sub-cases depending on the format of the two labels involved in the transitions.

Case $\underline{a}=a$. There exist $\hat{Q}, \hat{Q}^{\prime}, \tilde{q}$ with $Q \equiv(\boldsymbol{\nu} \tilde{q}) \hat{Q}$ and $Q^{\prime} \equiv(\boldsymbol{\nu} \tilde{q}) \hat{Q}^{\prime}$ such that:

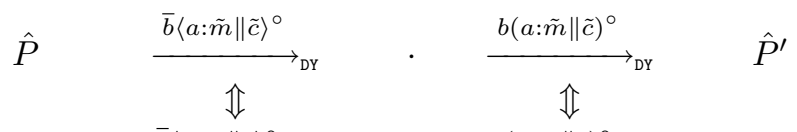

$$
\begin{aligned}
& \hat{P} \quad \stackrel{\bar{b}\langle a: \tilde{m} \| \tilde{c}\rangle^{\circ}}{\Downarrow}{ }_{\text {мтм }} \cdot \frac{b(a: \tilde{m} \| \tilde{c})^{\circ}}{\Downarrow}{ }_{\text {мтм }} \quad \hat{P}^{\prime} \\
& \hat{P} \quad \stackrel{(i) \dagger \bar{b}\langle a: \tilde{n} \| \tilde{c}\rangle_{i}^{\circ}}{\longmapsto_{\text {IIM }}} \quad . \quad \stackrel{(i)}{\longmapsto}_{\Downarrow \text { MTM }} \quad \hat{P}^{\prime} \quad(\tilde{n}=\tilde{m} \vee \tilde{n}=\tilde{c})
\end{aligned}
$$

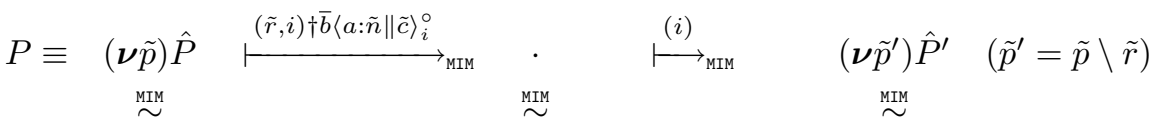

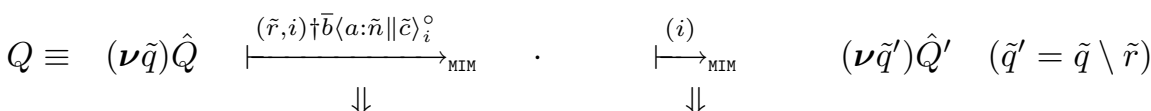

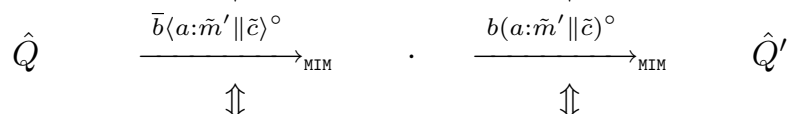

$$
\begin{aligned}
& \hat{Q} \quad \stackrel{\bar{b}\left\langle a: \tilde{m}^{\prime} \| \tilde{c}\right\rangle^{\circ}}{\longrightarrow} \mathrm{DY} \quad \cdot \stackrel{b\left(a: \tilde{m}^{\prime} \| \tilde{c}\right)^{\circ}}{\longrightarrow}{ }_{\mathrm{DY}} \quad \hat{Q}^{\prime}
\end{aligned}
$$

Thus, $Q \stackrel{\tau}{\longmapsto}$ DY $Q^{\prime}$, with $\left(\boldsymbol{\nu} \tilde{p}^{\prime}\right) \hat{P}^{\prime} \stackrel{\text { MIM }}{\sim}\left(\boldsymbol{\nu} \tilde{q}^{\prime}\right) \hat{Q}^{\prime}$. From this, by closure under restriction, we obtain $P^{\prime} \equiv\left(\boldsymbol{\nu} \tilde{p}^{\prime}, \tilde{r}\right) \hat{P}^{\prime} \stackrel{\text { MIM }}{\sim}\left(\boldsymbol{\nu} \tilde{q}^{\prime}, \tilde{r}\right) \hat{Q}^{\prime} \equiv Q^{\prime}$, as desired.

Case $\underline{a}=-$. There exist $\hat{Q}, \hat{Q}^{\prime}, \tilde{q}$ with $Q \equiv(\boldsymbol{\nu} \tilde{q}) \hat{Q}$ and $Q^{\prime} \equiv(\boldsymbol{\nu} \tilde{q}) \hat{Q}^{\prime}$ such that:

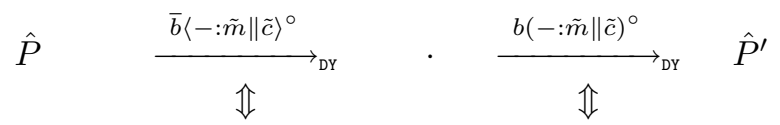

$$
\begin{aligned}
& \hat{P} \quad \stackrel{\bar{b}\langle-: \tilde{m} \| \tilde{c}\rangle^{\circ}}{\Downarrow} \longrightarrow_{\text {MIM }} \quad \cdot \frac{b(-: \tilde{m} \| \tilde{c})^{\circ}}{\Downarrow}{ }_{\text {MIM }} \hat{P}^{\prime}
\end{aligned}
$$

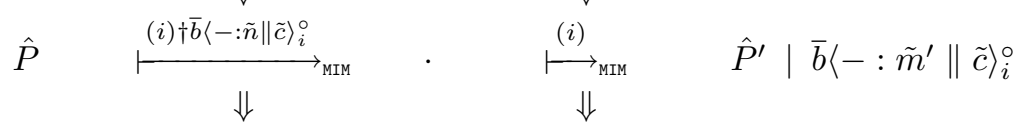

$$
\begin{aligned}
& P \equiv(\boldsymbol{\nu} \tilde{p}) \hat{P} \quad \stackrel{(\tilde{r}, i) \dagger \bar{b}<-: \tilde{n} \| \tilde{c}\rangle_{i}^{\circ}}{\longmapsto} \text { MIM } \quad . \quad \stackrel{(i)}{\longmapsto}_{\text {MIM }} \quad\left(\boldsymbol{\nu} \tilde{p}^{\prime}\right)\left(\hat{P}^{\prime} \mid \bar{b}\left\langle-: \tilde{m}^{\prime} \| \tilde{c}\right\rangle_{i}^{\circ}\right) \\
& \stackrel{\text { MTM }}{\sim} \stackrel{\text { MTM }}{\sim} \stackrel{\text { MTM }}{\sim}
\end{aligned}
$$

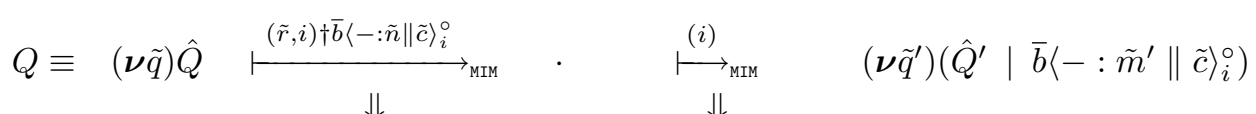

$$
\begin{aligned}
& \hat{Q} \quad \frac{\bar{b}\left\langle-: \tilde{m}^{\prime} \| \tilde{c}\right\rangle^{\circ}}{\mathfrak{i}}{ }_{\mathrm{MIM}} \cdot \frac{b\left(-: \tilde{m}^{\prime} \| \tilde{c}\right)^{\circ}}{\mathfrak{\Perp}}{ }_{\mathrm{MIM}} \hat{Q}^{\prime} \\
& \hat{Q} \quad \stackrel{\bar{b}\left\langle-: \tilde{m}^{\prime} \| \tilde{c}\right\rangle^{\circ}}{\longrightarrow} \mathrm{DY} \quad \cdot \stackrel{b\left(-: \tilde{m}^{\prime} \| \tilde{c}\right)^{\circ}}{\longrightarrow} \mathrm{DY} \quad \hat{Q}^{\prime}
\end{aligned}
$$


with $\tilde{p}^{\prime}=\tilde{p} \backslash \tilde{r}$ and $\tilde{q}^{\prime}=\tilde{q} \backslash \tilde{r}$. Thus, $Q \stackrel{\tau}{\longmapsto}$ DY $Q^{\prime}$ with $\left(\boldsymbol{\nu} \tilde{p}^{\prime}\right)\left(\hat{P}^{\prime} \mid \bar{b}\left\langle-: \tilde{m}^{\prime} \| \tilde{c}\right\rangle_{i}^{\circ} \stackrel{\text { MiM }}{\sim}\right.$ $\left(\boldsymbol{\nu} \tilde{q}^{\prime}\right)\left(\hat{Q}^{\prime} \mid \bar{b}\left\langle-: \tilde{m}^{\prime} \| \tilde{c}\right\rangle_{i}^{\circ}\right)$. By Lemma 5.1 we have that $\left(\boldsymbol{\nu} \tilde{p}^{\prime}\right) \hat{P}^{\prime} \stackrel{\text { MTM }}{\sim}\left(\boldsymbol{\nu} \tilde{q}^{\prime}\right) \hat{Q}^{\prime}$. Then, $P^{\prime} \stackrel{{ }^{\prime} \text { TIM }}{\sim} Q^{\prime}$ follows by closure under restriction with the names in $\tilde{r}$.

The hypothesis that $P$ and $Q$ are compatible processes is crucial for the proof. Indeed, the result is false for arbitrary run-time configurations. For instance $\bar{b}\langle-: m \| m\rangle_{i} \stackrel{\text { MTM }}{\sim} \mathbf{0}$, as neither process has any transition; on the other hand, clearly, $\bar{b}\langle-: m \| m\rangle_{i}{ }^{\text {D }} \mathbf{0}$ as the process on the left has an (i)-transition, while $\mathbf{0}$ clearly has not.

In order to prove the other inclusion, we first extend Lemma 4.2(3) to the case of secret and non-authentic messages, for the trusted MIM-derivatives. Intuitively, MIM-transitions never produce replicas of an indexed output, even in the case of non-authentic communication.

Lemma 6.4. Let $P$ be a MIM trusted derivative. If $P \equiv(\boldsymbol{\nu} \tilde{p})\left(\hat{P} \mid \bar{b}\langle-: \tilde{m} \| \tilde{c}\rangle_{i}^{\bullet}\right)$ then $\hat{P} \chi_{\tilde{c}}$.

Proof. The proof follows by the same argument used in Lemma 4.2 item 3.

Theorem 6.4. On trusted processes $\stackrel{\text { Dx }}{\sim} \subseteq \stackrel{\text { MIM }}{\sim}$.

Proof. We show that the following relation is a MIM-bisimulation:

$\mathscr{R}=\{(P, Q) \mid P \stackrel{\mathrm{D} \mathcal{R}}{\sim} Q$, with $P, Q$ trusted MIM-derivatives and compatible $\}$.

Let $(P, Q) \in \mathscr{R}$ and $P \stackrel{\alpha}{\longmapsto_{\text {MIM }}} P^{\prime}$ : we must find a matching transition $Q \stackrel{\alpha}{\longmapsto_{\text {MIM }}} Q^{\prime}$ with $P^{\prime} \stackrel{\text { DY }}{\sim} Q^{\prime}$. Clearly, $P^{\prime}$ and $Q^{\prime}$ are trusted MIM-derivatives and the fact that $P^{\prime}$ and $Q^{\prime}$ are compatible directly derive from Lemma 6.1 . We proceed by cases depending on the format of $\alpha$ (noting that $\alpha \neq \tau$ as there are no MIM-silent transitions in a trusted process).

If $\alpha \neq(i)$ we reason as in Theorem 6.3, namely:

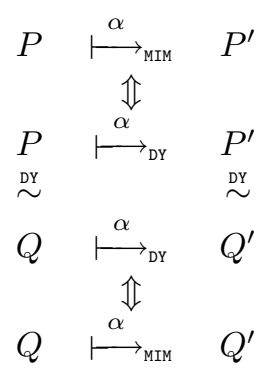

Let then $\alpha=(i)$. From $P \stackrel{(i)}{\longmapsto_{\text {MIM }}} P^{\prime}$ we know that $P \equiv(\boldsymbol{\nu} \tilde{p})\left(\hat{P} \mid \bar{b}\langle\underline{a}: \tilde{m} \| \tilde{c}\rangle_{i}^{\circ}\right)$. By Lemma 4.2(2), it follows that $P \stackrel{(i)}{\longmapsto}$ DY $P^{*} \equiv(\boldsymbol{\nu} \tilde{p})\left(\hat{P} \mid \bar{b}\langle\underline{a}: \tilde{m} \| \tilde{c}\rangle^{\circ}\right)$. Furthermore, an inspection of the labelled transition systems shows that $P \stackrel{(i)}{\longmapsto}_{\text {мI }} P^{\prime}$ implies $P \stackrel{(i)}{\longmapsto}_{\mathrm{DY}} P^{*} \stackrel{\tau}{\longmapsto}_{\mathrm{DY}} P^{\prime}$ where $\tau$ derives from the internal transitions $\hat{P} \mid \bar{b}\langle\underline{a}: \tilde{m} \| \tilde{c}\rangle^{\circ} \stackrel{\bar{b}\langle\underline{a}: \tilde{m} \| \tilde{c}\rangle^{\circ}}{\longrightarrow} \mathrm{DY} . \stackrel{b(\underline{a}: \tilde{m} \| \tilde{c})^{\circ}}{\longrightarrow}{ }_{\mathrm{DY}} \hat{P}^{\prime}$, with $P^{\prime} \equiv(\boldsymbol{\nu} \tilde{p}) \hat{P}^{\prime}$. We distinguish various sub-cases, depending on the format of these labels.

Case $\underline{a}=a$. We work out the sub-case when $\circ=\bullet$, the case when $\circ=\varepsilon$ follows by the same argument. Let then $P \equiv(\boldsymbol{\nu} \tilde{p})\left(\hat{P} \mid \bar{b}\langle a: \tilde{m} \| \tilde{c}\rangle_{i}^{\bullet}\right)$. From the hypothesis $P \stackrel{\text { Dr }}{\sim} Q$, and 
the observation that $P \stackrel{(i)}{\longmapsto_{\mathrm{DY}}} \cdot \stackrel{\tau}{\longmapsto}{ }_{\mathrm{DY}} P^{\prime}$, we know that $Q \stackrel{(i)}{\longmapsto}{ }_{\mathrm{DY}} Q^{*} \stackrel{\tau}{\longmapsto_{\mathrm{DY}}} Q^{\prime}$ with $P^{\prime} \stackrel{\mathrm{DY}}{\sim} Q^{\prime}$. To conclude, we need to show that $Q \stackrel{(i)}{\longmapsto}$ MIM $Q^{\prime}$. First, from the hypothesis that $P$ and $Q$ are compatible, it follows that $Q \equiv(\boldsymbol{\nu} \tilde{q})\left(\hat{Q} \mid \bar{b}\left\langle a: \tilde{m}^{\prime} \| \tilde{c}\right\rangle_{i}^{\bullet}\right)$. Then, by Lemma 4.2(2), it follows that $Q^{*} \equiv(\boldsymbol{\nu} \tilde{q})\left(\hat{Q} \mid \bar{b}\left\langle a: \tilde{m}^{\prime} \| \tilde{c}\right\rangle \bullet\right)$. Now we proceed by contradiction and assume that $Q \stackrel{(i)}{H^{\prime}}$ MIM $Q^{\prime}$. Then, the $\tau$-transition in $Q^{*} \stackrel{\tau}{\longmapsto}{ }_{\text {DY }} Q^{\prime}$ does not consume the output emitted by $Q \stackrel{(i)}{\longmapsto}$ DY , i.e. $Q^{\prime} \equiv(\boldsymbol{\nu} \tilde{q})\left(\hat{Q}^{\prime} \mid \bar{b}\left\langle a: \tilde{m}^{\prime} \| \tilde{c}\right\rangle^{\bullet}\right)$ which implies $Q^{\prime} \stackrel{\dagger(j) \bar{b}\left\langle a: \tilde{c} \| \tilde{c}{ }_{j}^{\bullet}\right.}{\longmapsto}$. On the other hand, by Lemma 4.2(3), $P^{\prime} \stackrel{\dagger(j) \bar{b}\langle a: \tilde{c} \| \tilde{c}\rangle_{j}^{\bullet}}{\longrightarrow}$ DY ${ }_{\mathrm{DY}}$ contradicting $P^{\prime} \stackrel{\mathrm{DP}}{\sim} Q^{\prime}$.

Case $\underline{a}=-$. We further distinguish two sub-cases, depending on whether the indexed message is in clear or encrypted.

We first examine the case $\circ=\bullet$. Then $P \equiv(\boldsymbol{\nu} \tilde{p})\left(\hat{P} \mid \bar{b}\langle-: \tilde{m} \| \tilde{c}\rangle_{i}^{\bullet}\right)$, and reasoning as in case $\underline{a}=a$, it follows that $Q \equiv(\boldsymbol{\nu} \tilde{q})\left(\hat{Q} \mid \bar{b}\left\langle-: \tilde{m}^{\prime} \| \tilde{c}\right\rangle_{i}^{\bullet}\right)$. Now, by Lemma 6.4 , we know that

$$
\begin{aligned}
P & \equiv(\boldsymbol{\nu} \tilde{p})\left(\hat{P} \mid \bar{b}\langle-: \tilde{m} \| \tilde{c}\rangle_{i}^{\bullet}\right) \\
Q & \equiv(\boldsymbol{\nu} \tilde{q})\left(\hat{Q} \mid \bar{b}\left\langle-: \tilde{m}^{\prime} \| \tilde{c}\right\rangle_{i}^{\bullet}\right)
\end{aligned}
$$

with $\hat{P} \Varangle_{\tilde{c}}$ and $\hat{Q} \Varangle_{\tilde{c}}$. From $P \stackrel{(i)}{\longmapsto}{ }_{\text {мI }} P^{\prime}$ it follows that $P^{\prime} \equiv\left(\boldsymbol{\nu} \tilde{p}^{\prime}\right)\left(\hat{P}^{\prime} \mid \bar{b}\langle-: \tilde{m} \| \tilde{c}\rangle_{i}^{\bullet}\right)$ with $\hat{P}^{\prime} \chi_{\tilde{c}}$. Again, reasoning as the the previous case, it must be the case that $Q \stackrel{(i)}{\longmapsto_{\text {MIM }}} Q^{\prime}$. Otherwise it would be $Q^{\prime} \equiv\left(\boldsymbol{\nu} \tilde{q}^{\prime}\right)\left(\hat{Q}^{\prime}\left|\bar{b}\langle-: \tilde{m} \| \tilde{c}\rangle_{i}^{\bullet}\right| \bar{b}\langle-: \tilde{m} \| \tilde{c}\rangle^{\bullet}\right)$ with $\hat{Q}^{\prime} \chi_{\tilde{c}}$, again contradicting $P^{\prime} \stackrel{\text { PY }}{\sim} Q^{\prime}$.

To conclude, assume $\circ=\varepsilon$. In this case, all names of the indexed message (including the $\tilde{m}$ 's) have been extruded at this stage, given that the communication is not secret and the message has already been intercepted (Lemma 4.2(2)). We have:

$$
\begin{aligned}
& P \underset{\Downarrow}{\stackrel{(i)}{\longmapsto}} \underset{\mathrm{DY}}{\Downarrow} \cdot \frac{\bar{b}\langle-: \tilde{m} \| \tilde{m}\rangle}{\Downarrow \mathrm{DY}} \cdot \frac{b(-: \tilde{m} \| \tilde{m})}{\Downarrow} P_{\mathrm{DY}} P^{\prime}
\end{aligned}
$$

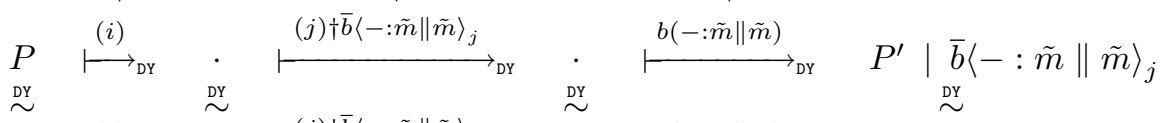

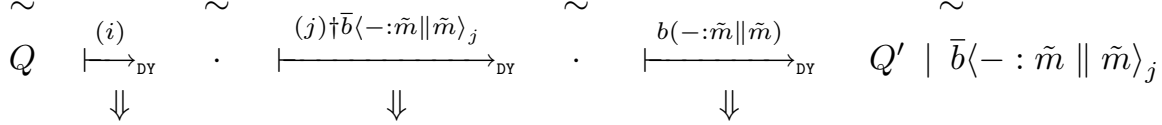

$$
\begin{aligned}
& Q \stackrel{(i)}{\longmapsto} \mathrm{DY} \quad . \quad \stackrel{\bar{b}\langle-: \tilde{m} \| \tilde{m}\rangle}{\longrightarrow} \mathrm{DY} \quad . \quad \stackrel{b(-: \tilde{m} \| \tilde{m})}{\longrightarrow} Q^{\prime}
\end{aligned}
$$

Since $P$ and $Q$ are compatible, By Lemma $4.2(2)$, we know that $Q \stackrel{(i)}{\longrightarrow}{ }_{\text {мтм }} Q^{\prime}$. From $P^{\prime}\left|\bar{b}\langle-: \tilde{m} \| \tilde{m}\rangle_{i} \stackrel{\text { DY }}{\sim} Q^{\prime}\right| \bar{b}\langle-: \tilde{m} \| \tilde{m}\rangle_{i}$, by Lemma 5.1, we derive $P^{\prime} \stackrel{\text { MIM }}{\sim} Q^{\prime}$, as desired.

By the previous two lemmas we have the result we anticipated.

Theorem 6.5. On trusted processes, $\stackrel{\text { MIM }}{\sim}=\stackrel{\text { DP }}{\sim}$. 
Besides being interesting in itself, as expressiveness result, Theorem 6.5 provides us with a very effective proof technique for $\stackrel{\mathrm{DY}}{\sim}$, and consequently for $\simeq$. In fact, coinductive proofs for MIM-bisimilarity may be carried out with much smaller candidates than their DY counterparts, as the number of states reached by a pair of trusted processes is much smaller in the MIM LTS than it is in the DY LTS. There are two reasons for that. First, trusted processes (and their derivatives) have no $\tau$ transitions in the MIM LTS (they do, instead, in the DY LTS). Secondly, the size of the candidate relations used in DY bisimilarity proof grows easily out of control due to the presence of multiple replicas of the same message. In contrast, MIM-transitions never produce replicas of an indexed output, as the index transitions are enabled only in processes that are ready to consume the produced outputs.

We show the use of MIM-bisimilarity as a proof technique for security in the next section.

\section{Security laws}

We discuss some equational laws that characterize the behavior of abstractions and provide insight into their (the abstractions') security properties. Mutatis mutandis, by combining the secrecy and authentication equations of this section one may derive coinductive proofs for the security properties of e-banking protocol in Section 3.1. Through this section we write $H \simeq K$ (respectively $H \sim K)$ to mean $[H] \simeq[K](\operatorname{resp} .[H] \sim[K])$.

\subsection{Secrecy and Authentication}

We start our security analysis by discussing the role of the scope restriction operator in our calculus. As we noted, restricting the destination of an output does not hide the presence of the output to an observer. Indeed, $(\boldsymbol{\nu} b) \bar{b}\langle\underline{a}: m\rangle \bullet \nsucc \mathbf{0}$, as outputs are always observable with an intercept, even when they are secret. On the other hand, secret outputs on restricted (or more generally trusted) channels do guarantee the privacy of the payload. This is expressed by the following equation:

$$
(\boldsymbol{\nu} b) \bar{b}\langle\underline{a}: m\rangle^{\bullet} \simeq(\boldsymbol{\nu} b) \bar{b}\left\langle\underline{a}: m^{\prime}\right\rangle^{\bullet}
$$

The equation is very easily proved by coinduction, using the following MIM-candidate, where $P$ and $Q$ are the trusted processes representing the two high-level principals in the equation:

$$
\mathscr{R}_{(1)}=\left\{(P, Q),\left(\bar{b}\langle\underline{a}: m \| c\rangle_{i}^{\bullet}, \bar{b}\left\langle\underline{a}: m^{\prime} \| c\right\rangle_{i}^{\bullet}\right)\right\}
$$

$\mathscr{R}_{(1)}$ works uniformly for the two cases, irrespective of whether $\underline{a}=a$ or $\underline{a}=-$. Notice, on the other hand, that the case $\underline{a}=-$ requires the following, significantly larger DYbisimulation:

$$
\left\{(P, Q),\left(\bar{b}\langle-: m \| c\rangle_{i}^{\bullet}, \bar{b}\left\langle-: m^{\prime} \| c\right\rangle_{i}^{\bullet}\right)\right\} \cup\left\{\left(\prod_{k} \bar{b}\langle-: m \| c\rangle^{\bullet}, \prod_{k} \bar{b}\left\langle-: m^{\prime} \| c\right\rangle^{\bullet}\right) \mid k \geq 0\right\}
$$

A variant of equation (1) uses a fresh name to masquerade for $m$ in place of $m^{\prime}$ : $(\boldsymbol{\nu} b) \bar{b}\langle\underline{a}: m\rangle^{\bullet} \simeq(\boldsymbol{\nu} b)(\boldsymbol{\nu} d) \bar{b}\langle\underline{a}: d\rangle^{\bullet}$, which is proved as the one we have just discussed. 
In (Abadi and Gordon, 1999), the spi-calculus characterization of secrecy is given by means of a related equation, that we may express as follows:

$$
(\boldsymbol{\nu} b)\left(\bar{b}\langle\underline{a}: m\rangle^{\bullet} \mid b(\underline{a}: x)^{\bullet} \cdot H(x)\right) \simeq(\boldsymbol{\nu} b)\left(\bar{b}\left\langle\underline{a}: m^{\prime}\right\rangle^{\bullet} \mid b(\underline{a}: x)^{\bullet} \cdot H(x)\right)
$$

which holds just in case $H(m) \simeq H\left(m^{\prime}\right)$ and, when $\underline{a}=-$, if $H(m), H\left(m^{\prime}\right)$ do not impersonate $b$. This last condition is to avoid $H(x)$ trivially breaks the secrecy of possible replays of $m$ and $m^{\prime}$ as in $H(x)=b(-: x)^{\bullet} \cdot \bar{c}\langle-: x\rangle$. The proof is similar to the proof of equation (1). Here we use the following MIM-bisimulation candidate.

$$
\mathscr{R}_{(2)}=\left\{(P, Q),\left(P_{i}, Q_{i}\right)\right\} \cup \sim
$$

$P$ and $Q$ are the trusted processes corresponding to the high-level principals in the equation, while $P_{i}$ and $Q_{i}$ are the residuals of the output intercepted transitions in the two processes, namely $\bar{b}\langle\underline{a}: m \| c\rangle_{i}^{\bullet} \mid b(a: x)^{\bullet} . H(x)$ and $\bar{b}\left\langle\underline{a}: m^{\prime} \| c\right\rangle_{i}^{\bullet} \mid b(a: x)^{\bullet} . H(x)$, respectively. For the non-authentic case, i.e., when $\underline{a}=-$, we additionally notice that

$$
\bar{b}\langle-: m \| c\rangle_{i}^{\bullet}\left|H(m) \sim \bar{b}\left\langle-: m^{\prime} \| c\right\rangle_{i}^{\bullet}\right| H\left(m^{\prime}\right)
$$

The above processes are reached from $P_{i}, Q_{i}$ after a replay of the intercepted output. The fact they are bisimilar is a simple consequence of $H(m)$ and $H\left(m^{\prime}\right)$ not impersonating $b$ : rule (Co-replay) of MIM semantics requires an input on $b$ to replay a message, thus the presence of $\bar{b}\langle-: m \| c\rangle_{i}^{\bullet}$ does not affect the behaviour of $H(m)$ in any way. Formally, the MIM-bisimulation candidate is:

$$
\mathscr{R}_{(3)}=\left\{\left(\bar{b}\langle-: m \| c\rangle_{i}^{\bullet}\left|P, \bar{b}\langle-: m \| c\rangle_{i}^{\bullet}\right| Q\right) . P \sim Q, H(m) \longmapsto_{\text {MIM }}^{*} P, H\left(m^{\prime}\right) \longmapsto_{\text {MIM }}^{*} Q\right\}
$$

In fact, from $H(m) \longmapsto{ }_{\text {MIM }}^{*} P$ and $H\left(m^{\prime}\right) \longmapsto{ }_{\text {MIM }}^{*} Q$ and since $H(m), H\left(m^{\prime}\right)$ do not impersonate $b$, we know that $P$ and $Q$ do not impersonate $b$. Thus, $\bar{b}\langle-: m \| c\rangle_{i}^{\bullet} \mid P \longmapsto^{\alpha}{ }_{\text {мтм }} P^{\prime}$ implies $P^{\prime}$ is $\bar{b}\langle-: m \| c\rangle_{i}^{\bullet} \mid P^{\prime \prime}$ and $P \stackrel{\alpha}{\longmapsto_{\text {MIM }}} P^{\prime \prime}$. The fact $\mathscr{R}_{(3)}$ is a MIM-bisimulation trivially follows from $P \sim Q$.

\subsection{Authentication}

The most basic form of authentication can be stated in terms of the equation $(\boldsymbol{\nu} a)(b(a$ : $\left.x)^{\circ} . P\right) \simeq \mathbf{0}$, which may be proved by just observing that neither process has any observational transition. A more interesting notion of authentication may be formalized as proposed by (Abadi and Gordon, 1999), by contrasting the system to be authenticated with a system that satisfies the specification trivially. To illustrate, consider the following equation:

$$
(\boldsymbol{\nu} a)\left(\bar{b}\langle a: m\rangle^{\circ} \mid b(a: x)^{\circ} . H(x)\right) \simeq(\boldsymbol{\nu} a)\left(\bar{b}\langle a: m\rangle^{\circ} \mid b(a: x)^{\circ} \cdot H(m)\right)
$$

Here, by "magically" plugging $m$ in $H(x)$, the equation states that $m$ is the only message that can possibly be received. That is guaranteed because there is just one authentic output in the scope of the restriction. The proof of equation (4) follows co-inductively showing that the following candidate is a MIM-bisimulation:

$$
\mathscr{R}_{(4)}=\left\{(P, Q),\left(P_{i}, Q_{i}\right)\right\} \cup \mathbf{I d}
$$


Here Id is the identity relation, $P$ and $Q$ are the trusted network processes corresponding to the high-level principals in the equation, while $P_{i}$ and $Q_{i}$ are the residuals of the output intercepted transitions in the two processes.

\subsection{Sessions}

We conclude our series of examples proving the authenticity and secrecy of properties of a simple protocol for establishing a private session between two communication parties. The specification is given by the following definitions:

$$
\begin{aligned}
D(m) & \stackrel{\text { def }}{=}(A(m) \mid B) \\
A(y) & \stackrel{\text { def }}{=}(\boldsymbol{\nu} k)\left(\bar{b}\langle a: k\rangle \mid a(b: x) \cdot \bar{x}\langle k: y\rangle^{\bullet}\right) \\
B & \stackrel{\text { def }}{=}(\boldsymbol{\nu} h) b(a: y) \cdot\left(\bar{a}\langle b: h\rangle \mid h(y: z)^{\bullet} \cdot H(z)\right)
\end{aligned}
$$

The two parties, $A$ and $B$, exchange two fresh names, $h$ and $k$, that are subsequently used for a secret and authentic exchange of the message $m$. The two fresh names $h$ and $k$ are thus employed to establish a new session between $A$ and $B$. To reason about authentication, let

$$
B^{\text {spec }}\left(z^{\prime}\right) \stackrel{\text { def }}{=}(\boldsymbol{\nu} h) b(a: y) \cdot\left(\bar{a}\langle b: h\rangle \mid h(y: z)^{\bullet} \cdot H\left(z^{\prime}\right)\right)
$$

represent the "ideal" definition of $B$, which differs from $B$ only in the fact that the received $z$ is ignored and, instead, $H$ gets the parameter $z^{\prime}$. In other words, $D^{\text {spec }}(m) \stackrel{\text { def }}{=}$ $\left(A(m) \mid B^{\text {spec }}(m)\right)$ represents a process which always delivers $m$ to $Q(z)$. The protocol properties may then be described as follows.

$$
\begin{aligned}
\text { (authenticity) } & D(m) \simeq D^{\text {spec }}(m) \\
(\text { secrecy) } & D(m) \simeq D\left(m^{\prime}\right) \text { if } H(m) \simeq H\left(m^{\prime}\right)
\end{aligned}
$$

The proof can be derived in essentially the same way for both equations: we give the proof for the secrecy equation as representative. While we could reason co-inductively, as for the previous equations, in this case it is more convenient to first show an auxiliary equation. Let:

$$
\begin{aligned}
A^{\prime}(y) & \stackrel{\text { def }}{=} \bar{b}\langle a: k\rangle \mid a(b: x) \cdot \bar{x}\langle k: y\rangle \\
B^{\prime} & \stackrel{\text { def }}{=} \quad b(a: y) \cdot\left(\bar{a}\langle b: h\rangle \mid h(y: z)^{\bullet} \cdot H(z)\right)
\end{aligned}
$$

We show, that $A^{\prime}(m)\left|B \simeq A^{\prime}\left(m^{\prime}\right)\right| B$. Then the proof of our initial equation derives by compositionality as $A^{\prime}(m)\left|B \simeq A^{\prime}\left(m^{\prime}\right)\right| B$ implies $(\boldsymbol{\nu} h)(\boldsymbol{\nu} k)\left(A^{\prime}(m) \mid B\right) \simeq$ $(\boldsymbol{\nu} h)(\boldsymbol{\nu} h)\left(A^{\prime}\left(m^{\prime}\right) \mid B\right)$ by closure under restriction and hence $A(m)\left|B \simeq A\left(m^{\prime}\right)\right| B$ because $A(x) \mid B \equiv(\boldsymbol{\nu} h)(\boldsymbol{\nu} k)\left(A^{\prime}(x) \mid B\right)$.

The proof that $A^{\prime}(m)\left|B \simeq A^{\prime}\left(m^{\prime}\right)\right| B$ follows by co-induction, choosing the candidate 
as follows. First define:

$$
\begin{aligned}
\mathscr{R}_{\text {sec }}= & \left\{\left(A^{\prime}(m)\left|B, A^{\prime}\left(m^{\prime}\right)\right| B\right),\right. \\
& \left(a(b: x) \cdot \bar{x}\langle k: m\rangle^{\bullet}|\bar{a}\langle b: h\rangle| h(k: z)^{\bullet} \cdot H(z),\right. \\
& \left.a(b: x) \cdot \bar{x}\left\langle k: m^{\prime}\right\rangle \bullet|\bar{a}\langle b: h\rangle| h(k: z)^{\bullet} \cdot H(z)\right), \\
& \left.\left(\bar{h}\langle k: m\rangle^{\bullet}\left|h(k: z)^{\bullet} \cdot H(z), \bar{h}\left\langle k: m^{\prime}\right\rangle^{\bullet}\right| h(k: z)^{\bullet} \cdot H(z)\right)\right\} \cup \simeq
\end{aligned}
$$

We can show that $\mathscr{R}_{\text {sec }}$ is a DY-bisimulation. The proof is routine, noting that some of the pairs that arise from $\mathscr{R}$ in the bisimulation game are contained in $\simeq$. One such pair is $\left(\left[a(b: x) . \bar{x}\langle k: m\rangle \bullet \mid B^{\prime}\right],\left[a(b: x) \cdot \bar{x}\left\langle k: m^{\prime}\right\rangle \bullet \mid B^{\prime}\right]\right)$, which arises from $\mathscr{R}$ via an (Output) transition, and is contained in $\simeq$ as both processes are stuck.

\section{Conclusions}

We have investigated a new set of security abstractions for distributed communication. The resulting primitives can be understood as a kernel API (Application Programming Interface) for the development of distributed applications. The API primitives are purposely defined without explicit reference to an implementation; at the same time, however, they are designed to be amenable to cryptographic implementations. The semantic theory and the proof techniques we have developed make the API a convenient tool for the analysis of security-sensitive applications. Our results show that the abstractions are robust, in that the observational equivalences they yield are preserved under the different observations available with the different adversarial primitives and interaction models which we have considered.

Certainly, for programming/specifying realistic examples and applications, one would need reliable communications within protected environments (a.k.a. secret channels à la pi-calculus). We do not see any problem in accommodating that feature within our present framework. Also, in its present form, our framework is targeted at (and we argue, well-suited for) secrecy and authentication. Future work includes expending it to account for advanced properties, like anonymity, required in modern network applications such as electronic voting.

Various papers in the literature have inspired or are related to our present approach. A localized use of names, introduced in the Local pi-calculus (Merro and Sangiorgi, 1998) is discussed and employed in (Abadi et al., 2002) for purposes similar to ours, while the handling of principals and authentication we adopted in the present paper is reminiscent of that in (Abadi et al., 2000). Other papers with related design are (Abadi and Fournet, 2004; Laud, 2005; Adão and Fournet, 2006). Of these, the closest to our approach is (Adão and Fournet, 2006). While we share some of the initial motivations and ideas, specifically the idea that the environment can mediate all communications, the two target complementary objectives, and differ for a number of design choices and technical results. A first important difference is in the choice of the communication primitives and their semantics: while we accommodate various communication modes, the semantics of communication in (Adão and Fournet, 2006) makes it possible to only express (what corresponds to) our secure communications. As a result, our calculus makes it possible 
to express a wider range of protocols. A second important difference is that we allow dynamic creation of new principal identities, thus making it possible to express sessions, a feature that is not easily accounted for in (Adão and Fournet, 2006).

Acknowledgements. We would like to thank the the referees for their comments and constructive criticism.

\section{References}

Abadi, M. (1998). Protection in programming-language translations. In Larsen, K. G., Skyum, S., and Winskel, G., editors, ICALP, volume 1443 of Lecture Notes in Computer Science, pages $868-883$. Springer.

Abadi, M. and Fournet, C. (2001). Mobile values, new names, and secure communication. In POPL 2001: The 28th ACM SIGPLAN-SIGACT Symposium on Principles of Programming Languages, London, pages 104-115.

Abadi, M. and Fournet, C. (2004). Private authentication. Theor. Comput. Sci., 322(3):427-476.

Abadi, M., Fournet, C., and Gonthier, G. (2000). Authentication primitives and their compilation. In POPL 2000, Proceedings of the 27th ACM SIGPLAN-SIGACT on Principles of Programming Languages, January 19-21, 2000, Boston, Massachusetts, USA, pages 302-315.

Abadi, M., Fournet, C., and Gonthier, G. (2002). Secure implementation of channel abstractions. Inf. Comput., 174(1):37-83.

Abadi, M. and Gordon, A. D. (1999). A calculus for cryptographic protocols: The spi calculus. Inf. Comput., 148(1):1-70.

Adão, P. and Fournet, C. (2006). Cryptographically sound implementations for communicating processes. In Bugliesi, M., Preneel, B., Sassone, V., and Wegener, I., editors, ICALP (2), volume 4052 of Lecture Notes in Computer Science, pages 83-94. Springer.

Bugliesi, M. and Focardi, R. (2008). Language based secure communication. In Proceedings of the 21st IEEE Computer Security Foundations Symposium, CSF 2008, Pittsburgh, Pennsylvania, 23-25 June 2008, pages 3-16. IEEE Computer Society.

Bugliesi, M. and Focardi, R. (2009). Security abstractions and intruder models. In Proceedings of the 15th Workshop on Expressiveness in Concurrency (EXPRESS 2008), number 242 in ENTCS, pages 99-112. Elsevier.

Corin, R., Deniélou, P.-M., Fournet, C., Bhargavan, K., and Leifer, J. J. (2007). Secure implementations for typed session abstractions. In 20th IEEE Computer Security Foundations Symposium, CSF 2007, 6-8 July 2007, Venice, Italy, pages 170-186. IEEE Computer Society.

Fournet, C. and Rezk, T. (2008). Cryptographically sound implementations for typed information-flow security. In Proceedings of the 35th ACM SIGPLAN-SIGACT Symposium on Principles of Programming Languages, POPL 2008, San Francisco, California, USA, January 7-12, 2008, pages 323-335. ACM.

Honda, K. and Yoshida, N. (1995). On reduction-based process semantics. Theor. Comput. Sci., 151(2):437-486.

Laud, P. (2005). Secrecy types for a simulatable cryptographic library. In Atluri, V., Meadows, C., and Juels, A., editors, ACM Conference on Computer and Communications Security, pages 26-35. ACM.

Merro, M. and Sangiorgi, D. (1998). On asynchrony in name-passing calculi. In Proceedings of ICALP 98, volume 1443 of Lecture Notes in Computer Science. Springer-Verlag. 
Merro, M. and Sangiorgi, D. (2004). On asynchrony in name-passing calculi. Mathematical Structures in Computer Science, 14(5):715-767.

Milner, R., Parrow, J., and Walker, D. (1992). A calculus of mobile processes, Parts I and II. Information and Computation, 100:1-77.

\section{Appendix A. Additional Proofs}

Lemma (6.1). Let $P$ and $Q$ be compatible processes. If $P \stackrel{\alpha}{\longmapsto} \eta P^{\prime}$ and $Q \stackrel{\alpha}{\longmapsto} \eta Q^{\prime}$, then $P^{\prime}$ and $Q^{\prime}$ are compatible, with $\eta$ any of DY and MIM.

Proof. By induction on the number of indexed messages in $P$. If $P$ does not have any indexed message, then neither does $Q$. Consequently, the only relevant transitions are the $(\cdots$ Intercepted) rules, whose side-conditions imply the claim.

Otherwise $P \equiv(\boldsymbol{\nu} \tilde{p})\left(\hat{P} \mid \bar{b}\langle\underline{a}: \tilde{m} \| \tilde{c}\rangle_{i}^{\circ}\right)$, and $Q \equiv(\boldsymbol{\nu} \tilde{q})\left(\hat{Q} \mid \bar{b}\left\langle\underline{a}: \tilde{m}^{\prime} \| \tilde{c}\right\rangle_{i}^{\circ}\right)$, with $\tilde{m}=\tilde{m}^{\prime}$ whenever $\circ=\varepsilon$ or $b \in \mathbf{N}_{u}$. By Lemma 4.2(1), $i$ does not occur as index in $\hat{P}$ and $\hat{Q}$. Then, we reason by a case analysis of the transitions, uniformly for the two systems:

If $\alpha \neq(i)$ we know that the transitions have the form

$$
\begin{array}{lll}
P \equiv(\boldsymbol{\nu} \tilde{p})\left(\hat{P} \mid \bar{b}\langle\underline{a}: \tilde{m} \| \tilde{c}\rangle_{i}^{\circ}\right) & \stackrel{\alpha}{ }_{\eta} & \left(\boldsymbol{\nu} \tilde{p}^{\prime}\right)\left(\hat{P}^{\prime} \mid \bar{b}\langle\underline{a}: \tilde{m} \| \tilde{c}\rangle_{i}^{\circ}\right) \equiv P^{\prime} \\
Q \equiv(\boldsymbol{\nu} \tilde{q})\left(\hat{Q} \mid \bar{b}\left\langle\underline{a}: \tilde{m}^{\prime} \| \tilde{c}\right\rangle_{i}^{\circ}\right) & \stackrel{\alpha}{\longmapsto}_{\eta} & \left(\boldsymbol{\nu} \tilde{q}^{\prime}\right)\left(\hat{Q}^{\prime} \mid \bar{b}\left\langle\underline{a}: \tilde{m}^{\prime} \| \tilde{c}\right\rangle_{i}^{\circ}\right) \equiv Q^{\prime}
\end{array}
$$

In particular, $(\boldsymbol{\nu} \tilde{p}) \hat{P} \stackrel{\alpha}{\longmapsto}_{\eta}\left(\boldsymbol{\nu} \tilde{p}^{\prime}\right) \hat{P}^{\prime}$ and $(\boldsymbol{\nu} \tilde{q}) \hat{Q} \stackrel{\alpha}{\longmapsto}_{\eta}\left(\boldsymbol{\nu} \tilde{q}^{\prime}\right) \hat{Q}^{\prime}$. Now, by inductive hypothesis we know that $\left(\boldsymbol{\nu} \tilde{p}^{\prime}\right) \hat{P}^{\prime}$ and $\left(\boldsymbol{\nu} \tilde{q}^{\prime}\right) \hat{Q}^{\prime}$ are compatible and, by Lemma $4.2(2), \hat{P}^{\prime} X_{i}$ and $\hat{Q}^{\prime} \chi_{i}$. As a consequence, $\left(\boldsymbol{\nu} \tilde{p}^{\prime}\right)\left(\hat{P}^{\prime} \mid \bar{b}\langle\underline{a}: \tilde{m} \| \tilde{c}\rangle_{i}^{\circ}\right) \equiv P^{\prime}$ and $\left(\boldsymbol{\nu} \tilde{q}^{\prime}\right)\left(\hat{Q}^{\prime} \mid \bar{b}\left\langle\underline{a}: \tilde{m}^{\prime} \| \tilde{c}\right\rangle_{i}^{\circ}\right) \equiv$ $Q^{\prime}$ are also compatible.

If $\alpha=(i)$, i.e., the transition is a (co-forward) or a (co-reply), the effect is to either cancel the indexed copy, from $P$ and $Q$, or leave it untouched: in both cases $P^{\prime}$ and $Q^{\prime}$ are compatible.

Lemma (6.2). Let $\gamma_{i}=\bar{a}\langle\underline{b}: \tilde{m} \| \tilde{c}\rangle_{i}^{\circ}$ and $\gamma_{i}^{\prime}=\bar{a}\left\langle\underline{b}: \tilde{m}^{\prime} \| \tilde{c}\right\rangle_{i}^{\circ}$, and let $P_{i}$ and $Q_{i}$ be the two trusted derivatives $P_{i} \equiv(\boldsymbol{\nu} \tilde{p})\left(\hat{P} \mid \gamma_{i}\right)$ and $Q_{i} \equiv(\boldsymbol{\nu} \tilde{q})\left(\hat{Q} \mid \gamma_{i}^{\prime}\right)$. If $P_{i} \stackrel{\eta}{\sim} Q_{i}$ then, for any $j \notin f n\left(P_{i}, Q_{i}\right) \cup\{\tilde{p}, \tilde{q}\}$, we have:

$1 \quad P_{j} \equiv(\boldsymbol{\nu} \tilde{p})\left(\hat{P} \mid \gamma_{j}\right) \stackrel{\eta}{\sim}(\boldsymbol{\nu} \tilde{q})\left(\hat{Q} \mid \gamma_{j}^{\prime}\right) \equiv Q_{j}$

$2 \quad P_{i, j} \equiv(\boldsymbol{\nu} \tilde{p})\left(\hat{P}\left|\gamma_{i}\right| \gamma_{j}\right) \stackrel{\eta}{\sim}(\boldsymbol{\nu} \tilde{q})\left(\hat{Q}\left|\gamma_{i}^{\prime}\right| \gamma_{j}^{\prime}\right) \equiv Q_{i, j}$ when $\underline{b}=-$

where $\stackrel{\eta}{\sim}$ is either $\stackrel{\text { DY }}{\sim}$ or $\stackrel{\text { MIM }}{\sim}$, and $\gamma_{j}$ and $\gamma_{j}^{\prime}$ are $\gamma$ and $\gamma^{\prime}$ indexed by $j$ rather than $i$.

Proof. In both cases, the proof is by coinduction, uniformly for the two bisimilarities. First observe that, by Lemma 4.2(1,2) $\hat{P} \chi_{i, j}$, and similarly $\hat{Q} \chi_{i, j}$. Moreover, $i \neq j$, given that, again by Lemma 4.2(1), $i \in f n\left(P_{i}, Q_{i}\right)$.

For (1), we define $\mathscr{R}=\left\{\left(P_{j}, Q_{j}\right) \mid P_{i} \stackrel{\eta}{\sim} Q_{i}\right\} \cup \stackrel{\eta}{\sim}$ and show that $\mathscr{R}$ is an $\eta$-bisimulation. Take $\left(P_{j}, Q_{j}\right) \in \mathscr{R}$ and let $P_{j} \stackrel{\alpha}{\longmapsto}_{\eta} R$.

— If $\alpha \neq(j)$, given that $\hat{P} \chi_{i, j}$, we know that $R \equiv\left(\boldsymbol{\nu} \tilde{p}^{\prime}\right)\left(\hat{P}^{\prime} \mid \gamma_{j}\right)$, and also $P_{i} \stackrel{\alpha}{\longmapsto} P_{i}^{\prime} \equiv$ $\left(\boldsymbol{\nu} \tilde{p}^{\prime}\right)\left(\hat{P}^{\prime} \mid \gamma_{i}\right)$. From the hypothesis $P_{i} \stackrel{\eta}{\sim} Q_{i}$, we then have $Q_{i} \stackrel{\alpha}{\longmapsto} S$ with $R \stackrel{\eta}{\sim} S$. 
Since $\hat{Q} \chi_{i, j}$, it follows that $S \equiv\left(\boldsymbol{\nu} \tilde{q}^{\prime}\right)\left(\hat{Q}^{\prime} \mid \gamma_{i}^{\prime}\right)$, and also $Q_{j} \stackrel{\alpha}{\longmapsto}_{\eta} Q_{j}^{\prime} \equiv\left(\boldsymbol{\nu} \tilde{p}^{\prime}\right)\left(\hat{Q}^{\prime} \mid \gamma_{j}^{\prime}\right)$, which is the desired matching move from $Q_{j}$.

- If instead $\alpha=(j)$, we have four different cases, depending (i) on the labelled transition system under consideration (i.e. whether $\eta$ is DY or MIM) and (ii) on whether $\gamma_{i}$ and $\gamma_{i}^{\prime}$ are authentic or not.

We first look at the DY-system. If $\underline{b}=b$, then $P_{j} \stackrel{(j)}{\longmapsto}$ Dy $R \equiv(\boldsymbol{\nu} \tilde{p})(\hat{P} \mid \gamma)$ (where $\gamma$ is the output corresponding to $\left.\gamma_{i}\right)$ and given that $\hat{P} \chi_{i, j}$, it follows that $P_{i} \stackrel{(i)}{\longmapsto}$ Dy $R$. From the hypothesis $P_{i} \stackrel{\text { Dr }}{\sim} Q_{i}$, we then have $Q_{i} \stackrel{(i)}{\longmapsto_{\mathrm{Dr}}} S$ with $R \stackrel{\text { Dx }}{\sim} S$. Now, from $\hat{Q} \chi_{i, j}$, it follows that $S \equiv(\boldsymbol{\nu} \tilde{q})\left(\hat{Q} \mid \gamma^{\prime}\right)$, and also that $Q_{j} \stackrel{(j)}{\mathfrak{D x}_{\mathrm{r}}} S$ which is the matching we needed to conclude. If $\underline{b}=-$, we have $P_{j} \stackrel{(j)}{\longmapsto} R_{\mathrm{DY}} \equiv(\boldsymbol{\nu} \tilde{p})\left(\hat{P}|\gamma| \gamma_{j}\right)$, and $Q_{j} \stackrel{(j)}{\longmapsto}_{\mathrm{DY}} S_{j} \equiv(\boldsymbol{\nu} \tilde{q})\left(\hat{Q}\left|\gamma^{\prime}\right| \gamma_{j}^{\prime}\right)$, with $R_{i} \stackrel{\text { Dx }}{\sim} S_{i}$ given that $P_{i} \stackrel{(i)}{\longmapsto_{\mathrm{DY}}} R_{i}$ is necessarily simulated by $Q_{i} \stackrel{(i)}{\longmapsto}$ Dy $S_{i}$ being $\hat{Q} \chi_{i, j}$. Hence, $\left(R_{j}, S_{j}\right) \in \mathscr{R}$ as desired.

Now let's consider the MIM-system. If $\underline{b}=b$, then $P_{j} \stackrel{(j)}{\longmapsto_{\text {мтм }}} R \equiv(\boldsymbol{\nu} \tilde{p}) \hat{P}^{\prime}$, where $\hat{P} \stackrel{a(b: \tilde{m} \| \tilde{c})}{\longrightarrow}_{\longrightarrow} \hat{P}^{\prime}$ and a corresponding transition is available from $P_{i}$, i.e. $P_{i} \stackrel{(i)}{\longrightarrow}_{\text {мтм }} R$. From the hypothesis $P_{i} \stackrel{\text { MrM }}{\sim} Q_{i}$, we then have $Q_{i} \stackrel{(i)}{\longmapsto_{\text {MIM }}} S$ with $R \stackrel{\text { DP }}{\sim} S$. Now, from $\hat{Q} X_{i, j}$, it follows that that $Q_{j} \stackrel{(j)}{\longmapsto}$ итм $S$ which is the matching we needed to conclude. Finally, If $\underline{b}=-$, we have $P_{j} \stackrel{(j)}{\longmapsto}_{\text {MIM }} R_{j} \equiv(\boldsymbol{\nu} \tilde{p})\left(\hat{P}^{\prime} \mid \gamma_{j}\right)$, and $Q_{j} \stackrel{(j)}{\longmapsto}_{\text {MIM }} S_{j} \equiv(\boldsymbol{\nu} \tilde{q})\left(\hat{Q}^{\prime} \mid \gamma_{j}^{\prime}\right)$, with $R_{i} \stackrel{\text { MrM }}{\sim} S_{i}$ given that $P_{i} \stackrel{(i)}{\longmapsto}$ DY $R_{i}$ is necessarily simulated by $Q_{i} \stackrel{(i)}{\longmapsto}$ DY $S_{i}$ being $\hat{Q} \chi_{i, j}$. Hence $\left(R_{j}, S_{j}\right) \in \mathscr{R}$ as desired.

For (2), we define the candidate relation as follows: $\mathscr{R}=\left\{\left(P_{i, j}, Q_{i, j}\right) \mid P_{i} \stackrel{\eta}{\sim} Q_{i}\right\}$. Then we proceed by coinduction taking $\left(P_{i, j}, Q_{i, j}\right) \in \mathscr{R}$ and $P_{i, j} \longmapsto_{\eta} R$ and showing that there exists a matching transition for from $Q_{i, j}$. Notice that we are assuming that $\underline{b}=-$, i.e., that communication is not authenticated. This means that $\gamma_{i}, \gamma_{i}^{\prime}$ and $\gamma_{j}, \gamma_{j}^{\prime}$ will never be consumed by any transition. We distinguish two cases: if the $\alpha \neq(j)$ the move must come from $P_{i}$ and proof follows easily from the hypothesis that $P_{i} \stackrel{\eta}{\sim} Q_{i}$. The case $\alpha=(j)$ is just as easy because, by (1) we know that $P_{i} \stackrel{\eta}{\sim} Q_{i}$ iff $P_{j} \stackrel{\eta}{\sim} Q_{j}$ and we may reason as in previous case, i.e., $\alpha \neq(j)$, interchanging $j$ with $i$.

Lemma (6.3). Assume $P$ and $Q$ compatible. If $P \stackrel{\text { MIM }}{\sim} Q$ and $P \stackrel{(i)}{\longmapsto}$ DY $P^{\prime}$ then $Q \stackrel{(i)}{\longmapsto}$ DY $Q^{\prime}$

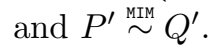

Proof. we show that the following relation is a MIM-bisimulation.

$$
\mathscr{R}=\left\{\left(P^{\prime}, Q^{\prime}\right) \mid \exists P, Q \text { compatible. } P \stackrel{(i)}{\longrightarrow} P_{\mathrm{DY}} P^{\prime}, Q \stackrel{(i)}{\longrightarrow}{ }_{\mathrm{DY}} Q^{\prime}, P \stackrel{\text { MIM }}{\sim} Q\right\} \cup \stackrel{\text { MIT }}{\sim}
$$

Assume $\left(P^{\prime}, Q^{\prime}\right) \in \mathscr{R}$ and $P^{\prime} \stackrel{\alpha}{\longmapsto}_{\text {мт }} P^{\prime \prime}$. From $\left(P^{\prime}, Q^{\prime}\right) \in \mathscr{R}$, we know that there exist $P$ and $Q$ compatible such that $P \stackrel{(i)}{\longmapsto}$ DY $P^{\prime}, Q \stackrel{(i)}{\longmapsto}$ DY $Q$ and $P \stackrel{\mathrm{DY}}{\sim} Q$. From $P \stackrel{(i)}{\longmapsto}$ DY , an inspection of the DY-transition system shows that $P \equiv(\boldsymbol{\nu} \tilde{p})\left(\hat{P} \mid \bar{b}\langle\underline{a}: \tilde{m} \| \tilde{c}\rangle_{i}^{\circ}\right)$. Then, 
since $P$ and $Q$ are compatible, it follows that $Q \equiv(\boldsymbol{\nu} \tilde{q})\left(\hat{Q} \mid \bar{b}\left\langle\underline{a}: \tilde{m}^{\prime} \| \tilde{c}\right\rangle_{i}^{\circ}\right)$. We now have two cases, depending on the value of $\underline{a}$.

We start with the case $\underline{a}=a$. By Lemma 4.2(2), the index $i$ is unique in $P$ and $Q$, hence we have $P^{\prime} \equiv(\boldsymbol{\nu} \tilde{p})\left(\hat{P} \mid \bar{b}\langle a: \tilde{m} \| \tilde{c}\rangle^{\circ}\right)$ and $Q^{\prime} \equiv(\boldsymbol{\nu} \tilde{q})\left(\hat{Q} \mid \bar{b}\left\langle a: \tilde{m}^{\prime} \| \tilde{c}\right\rangle^{\circ}\right)$. Now we examine the transition $P^{\prime} \stackrel{\alpha}{\longmapsto} \longmapsto_{\text {мт }} P^{\prime \prime}$, and distinguish three sub-cases:

— The move $\alpha$ is by process $\hat{P}$. From $\hat{P} \chi_{i}$, we know that $\alpha \neq(i)$, and we may reason as follows:

$$
\begin{aligned}
& P^{\prime} \equiv(\boldsymbol{\nu} \tilde{p})\left(\hat{P} \mid \bar{b}\langle a: \tilde{m} \| \tilde{c}\rangle^{\circ}\right) \quad \stackrel{\alpha}{\longmapsto_{\text {мтм }}}\left(\boldsymbol{\nu} \tilde{p}^{\prime}\right)\left(\hat{P}^{\prime} \mid \bar{b}\langle a: \tilde{m} \| \tilde{c}\rangle^{\circ}\right) \quad \equiv P^{\prime \prime} \\
& \Downarrow \\
& P \equiv(\boldsymbol{\nu} \tilde{p})\left(\hat{P} \mid \bar{b}\langle a: \tilde{m} \| \tilde{c}\rangle_{i}^{\circ}\right) \quad \stackrel{\alpha}{\longmapsto_{\text {MIM }}}\left(\boldsymbol{\nu} \tilde{p}^{\prime}\right)\left(\hat{P}^{\prime} \mid \bar{b}\langle a: \tilde{m} \| \tilde{c}\rangle_{i}^{\circ}\right) \quad \equiv P^{\prime \prime \prime} \\
& \stackrel{\text { MIM }}{\sim} \underset{\sim}{\sim} \\
& Q \equiv(\boldsymbol{\nu} \tilde{q})\left(\hat{Q} \mid \bar{b}\left\langle a: \tilde{m}^{\prime} \| \tilde{c}\right\rangle_{i}^{\circ}\right) \quad \stackrel{\alpha}{\longmapsto_{\text {тги }}}\left(\boldsymbol{\nu} \tilde{q}^{\prime}\right)\left(\hat{Q}^{\prime} \mid \bar{b}\left\langle a: \tilde{m}^{\prime} \| \tilde{c}\right\rangle_{i}^{\circ}\right) \equiv Q^{\prime \prime \prime} \\
& \Downarrow \\
& Q^{\prime} \equiv(\boldsymbol{\nu} \tilde{q})\left(\hat{Q} \mid \bar{b}\left\langle a: \tilde{m}^{\prime} \| \tilde{c}\right\rangle^{\circ}\right) \quad \stackrel{\alpha}{\longmapsto_{\text {мтм }}}\left(\boldsymbol{\nu} \tilde{q}^{\prime}\right)\left(\hat{Q}^{\prime} \mid \bar{b}\left\langle a: \tilde{m}^{\prime} \| \tilde{c}\right\rangle^{\circ}\right) \equiv Q^{\prime \prime}
\end{aligned}
$$

That $Q^{\prime \prime \prime} \equiv\left(\boldsymbol{\nu} \tilde{q}^{\prime}\right)\left(\hat{Q}^{\prime} \mid \bar{b}\left\langle a: \tilde{m}^{\prime} \| \tilde{c}\right\rangle_{i}^{\circ}\right)$ follows because the only move possible for $\bar{b}\left\langle a: \tilde{m}^{\prime} \| \tilde{c}\right\rangle_{i}^{\circ}$ is a forward labelled $(i)$, while $\alpha \neq(i)$ : hence the move from $Q$ must have originated from $\hat{Q}$. Thus, from $P^{\prime} \stackrel{\alpha}{\longmapsto}_{\text {MтM }} P^{\prime \prime}$ we have found a matching move $Q^{\prime} \stackrel{\alpha}{\longmapsto}_{\text {мті }} Q^{\prime \prime}$. Now we must show that $\left(P^{\prime \prime}, Q^{\prime \prime}\right) \in \mathscr{R}$. First observe that $P^{\prime \prime \prime}$ and $Q^{\prime \prime \prime}$ are compatible: this follows by Lemma 6.1, and from the hypothesis that $P$ and $Q$ are compatible. Also note that $P^{\prime \prime \prime} \stackrel{(i)}{\longmapsto}_{\mathrm{DY}} P^{\prime \prime}, Q^{\prime \prime \prime} \stackrel{(i)}{\longmapsto}_{\mathrm{DY}} Q^{\prime \prime}$. Then, $\left(P^{\prime \prime}, Q^{\prime \prime}\right) \in \mathscr{R}$ follows by definition, as $P^{\prime \prime \prime} \stackrel{\text { MTM }}{\sim} Q^{\prime \prime \prime}$ with $P^{\prime \prime \prime}$ and $Q^{\prime \prime \prime}$ compatible.

- The move $\alpha$ is by process $\bar{b}\langle a: \tilde{m} \| \tilde{c}\rangle^{\circ}$ and is an output. By an inspection of the observable transitions, we know that $b \in \mathbf{N}_{u}$ or $\circ=\varepsilon$ and the transition is of the following form:

$$
P^{\prime} \equiv(\boldsymbol{\nu} \tilde{p})\left(\hat{P} \mid \bar{b}\langle a: \tilde{m} \| \tilde{c}\rangle^{\circ}\right) \stackrel{\bar{b}\langle a: \tilde{m} \| \tilde{c}\rangle^{\circ}}{\longrightarrow} \text { мтм }(\boldsymbol{\nu} \tilde{p}) \hat{P} .
$$

Notice that no name gets extruded in this move. In fact, since $P \equiv(\boldsymbol{\nu} \tilde{p})\left(\hat{P} \mid \bar{b}\langle a: \tilde{m} \| \tilde{c}\rangle_{i}^{\circ}\right)$, by Lemma 4.2(1) we know that $i, b, \underline{a}, \tilde{c}, \tilde{m} \in f n(P)$. Back on $Q \equiv(\boldsymbol{\nu} \tilde{q})\left(\hat{Q} \mid \bar{b}\left\langle a: \tilde{m}^{\prime} \| \tilde{c}\right\rangle_{i}^{\circ}\right)$, the fact that $P$ and $Q$ are compatible, together with $b \notin \mathbf{N}_{t}$ imply that $\tilde{m}=\tilde{m}^{\prime}$. Thus we find the desired matching move from $Q^{\prime}$ :

$$
Q^{\prime} \equiv(\boldsymbol{\nu} \tilde{q})\left(\hat{Q} \mid \bar{b}\langle a: \tilde{m} \| \tilde{c}\rangle^{\circ}\right) \stackrel{\bar{b}\langle a: \tilde{m} \| \tilde{c}\rangle^{\circ}}{\longrightarrow} \text { мгм }(\boldsymbol{\nu} \tilde{q}) \hat{Q} .
$$

To conclude, by our hypothesis we know that $P \stackrel{\text { MTM }}{\sim} Q$, and this, by Lemma 5.1 implies $(\boldsymbol{\nu} \tilde{p}) \hat{P} \stackrel{\text { MTM }}{\sim}(\boldsymbol{\nu} \tilde{q}) \hat{Q}$, hence $(\boldsymbol{\nu} \tilde{p}) \hat{P} \mathscr{R}(\boldsymbol{\nu} \tilde{q}) \hat{Q}$ as desired. 
— The move $\alpha$ is by process $\bar{b}\langle a: \tilde{m} \| \tilde{c}\rangle^{\circ}$ and is an intercepted output. Consider the following reductions:

$$
\begin{aligned}
& P^{\prime} \equiv(\boldsymbol{\nu} \tilde{p})\left(\hat{P} \mid \bar{b}\langle a: \tilde{m} \| \tilde{c}\rangle^{\circ}\right) \quad \stackrel{(j)+\bar{b}\langle a: \tilde{n} \| \tilde{c}\rangle_{j}^{\circ}}{\longmapsto} \underset{\operatorname{MTM}}{\stackrel{\nu}{\nu^{2}}}(\tilde{p})\left(\hat{P} \mid \bar{b}\langle a: \tilde{m} \| \tilde{c}\rangle_{j}^{\circ}\right) \equiv P^{\prime \prime} \\
& Q^{\prime} \equiv(\boldsymbol{\nu} \tilde{q})\left(\hat{Q} \mid \bar{b}\left\langle a: \tilde{m}^{\prime} \| \tilde{c}\right\rangle^{\circ}\right) \quad \stackrel{(j) \dagger \bar{b}\langle a: \tilde{n} \| \tilde{c}\rangle_{j}^{\circ}}{\longmapsto} \stackrel{\text { MтM }}{~}^{\prime}(\boldsymbol{\nu} \tilde{q})\left(\hat{Q} \mid \bar{b}\left\langle a: \tilde{m}^{\prime} \| \tilde{c}\right\rangle_{j}^{\circ}\right) \equiv Q^{\prime \prime}
\end{aligned}
$$

where $j$ is a fresh index, and either $\tilde{n}=\tilde{c}$ (if $\circ=\bullet$ and $b \in \mathbf{N}_{t}$ ), or otherwise $\tilde{n}=\tilde{m}=\tilde{m}^{\prime}$, given that $P$ and $Q$ are compatible. Notice that, even in this case, no name gets extruded in this moves. Now, from our hypothesis $P \stackrel{\text { MTM }}{\sim} Q$, by Lemma 6.2(1) we obtain $P^{\prime \prime} \stackrel{\text { Mrin }}{\sim} Q^{\prime \prime}$, hence $P^{\prime \prime} \mathscr{R} Q^{\prime \prime}$ as desired.

We continue with the case $\underline{a}=-$. As in our previous analysis, by Lemma 4.2(2), we know that the index $i$ is unique in $P$ and $Q$. Given that the originator of the message is anonymous, the $(i)$ move from $P$ and $Q$ is a replay, and thus we have $P^{\prime} \equiv$ $(\boldsymbol{\nu} \tilde{p})\left(\hat{P}\left|\bar{b}\langle-: \tilde{m} \| \tilde{c}\rangle^{\circ}\right| \bar{b}\langle-: \tilde{m} \| \tilde{c}\rangle_{i}^{\circ}\right)$ and $Q^{\prime} \equiv(\boldsymbol{\nu} \tilde{q})\left(\hat{Q}\left|\bar{b}\left\langle-: \tilde{m}^{\prime} \| \tilde{c}\right\rangle^{\circ}\right| \bar{b}\left\langle-: \tilde{m}^{\prime} \| \tilde{c}\right\rangle_{i}^{\circ}\right)$. We examine the transition $P^{\prime} \stackrel{\alpha}{\longmapsto_{\text {мIM }}} P^{\prime \prime}$, and distinguish four sub-cases:

— The move $\alpha$ is by process $\hat{P}$. From $\hat{P} \chi_{i}$, we know that $\alpha \neq(i)$, and we may reason as follows:

$$
\begin{aligned}
& P^{\prime} \equiv(\boldsymbol{\nu} \tilde{p})\left(\hat{P}\left|\bar{b}\langle-: \tilde{m} \| \tilde{c}\rangle^{\circ}\right| \bar{b}\langle-: \tilde{m} \| \tilde{c}\rangle_{i}^{\circ}\right) \\
& \stackrel{\alpha}{\longmapsto} \text { мтм }\left(\nu \tilde{p}^{\prime}\right)\left(\hat{P}^{\prime}\left|\bar{b}\langle-: \tilde{m} \| \tilde{c}\rangle^{\circ}\right| \bar{b}\langle-: \tilde{m} \| \tilde{c}\rangle_{i}^{\circ}\right) \equiv P^{\prime \prime} \\
& \Downarrow \\
& P \equiv(\boldsymbol{\nu} \tilde{p})\left(\hat{P} \mid \bar{b}\langle-: \tilde{m} \| \tilde{c}\rangle_{i}^{\circ}\right) \stackrel{\alpha}{\stackrel{\alpha}{\sim}} \underset{\text { мгм }}{\sim}\left(\boldsymbol{\nu} \tilde{p}^{\prime}\right)\left(\hat{P}^{\prime} \mid \bar{b}\langle-: \tilde{m} \| \tilde{c}\rangle_{i}^{\circ}\right) \equiv P^{\prime \prime \prime} \\
& Q \equiv(\boldsymbol{\nu} \tilde{q})\left(\hat{Q} \mid \bar{b}\left\langle-: \tilde{m}^{\prime} \| \tilde{c}\right\rangle_{i}^{\circ}\right) \stackrel{\alpha}{\longmapsto}_{\text {мIM }}\left(\boldsymbol{\nu} \tilde{q}^{\prime}\right)\left(\hat{Q}^{\prime} \mid \bar{b}\left\langle-: \tilde{m}^{\prime} \| \tilde{c}\right\rangle_{i}^{\circ}\right) \equiv Q^{\prime \prime \prime} \\
& \Downarrow \\
& Q^{\prime} \equiv(\boldsymbol{\nu} \tilde{q})\left(\hat{Q}\left|\bar{b}\left\langle-: \tilde{m}^{\prime} \| \tilde{c}\right\rangle^{\circ}\right| \bar{b}\left\langle-: \tilde{m}^{\prime} \| \tilde{c}\right\rangle_{i}^{\circ}\right) \\
& \stackrel{\alpha}{\longmapsto} \text { MIM }\left(\nu \tilde{q}^{\prime}\right)\left(\hat{Q}^{\prime}\left|\bar{b}\left\langle-: \tilde{m}^{\prime} \| \tilde{c}\right\rangle^{\circ}\right| \bar{b}\left\langle-: \tilde{m}^{\prime} \| \tilde{c}\right\rangle_{i}^{\circ}\right) \equiv Q^{\prime \prime}
\end{aligned}
$$

That $Q^{\prime \prime \prime} \equiv\left(\boldsymbol{\nu} \tilde{q}^{\prime}\right)\left(\hat{Q}^{\prime} \mid \bar{b}\left\langle-: \tilde{m}^{\prime} \| \tilde{c}\right\rangle_{i}^{\circ}\right)$ follows because the only move possible for $\bar{b}\left\langle-: \tilde{m}^{\prime} \| \tilde{c}\right\rangle_{i}^{\circ}$ is a replay labelled $(i)$, while $\alpha \neq(i)$ : hence the move from $Q$ must have originated from $\hat{Q}$. Now we must show that $\left(P^{\prime \prime}, Q^{\prime \prime}\right) \in \mathscr{R}$. First we observe that $P^{\prime \prime \prime}$ and $Q^{\prime \prime \prime}$ are compatible: this follows by Lemma 6.1 , and from the hypothesis that $P$ and $Q$ are compatible. Then, we note that $P^{\prime \prime \prime} \stackrel{(i)}{\longmapsto}_{\mathrm{DY}} P^{\prime \prime}, Q^{\prime \prime \prime} \stackrel{(i)}{\longmapsto}_{\mathrm{DY}} Q^{\prime \prime}$. Finally, $\left(P^{\prime \prime}, Q^{\prime \prime}\right) \in \mathscr{R}$ follows by definition, as $P^{\prime \prime \prime} \stackrel{\text { MTM }}{\sim} Q^{\prime \prime \prime}$ with $P^{\prime \prime \prime}$ and $Q^{\prime \prime \prime}$ compatible. 
— The move $\alpha$ is by process $\bar{b}\langle-: \tilde{m} \| \tilde{c}\rangle_{i}^{\circ}$. The analysis of the transition is as follows:

$$
P^{\prime} \equiv(\boldsymbol{\nu} \tilde{p})\left(\hat{P}\left|\bar{b}\langle-: \tilde{m} \| \tilde{c}\rangle^{\circ}\right| \bar{b}\langle-: \tilde{m} \| \tilde{c}\rangle_{i}^{\circ}\right)
$$

$$
\stackrel{(i)}{\longmapsto}_{\text {МтM }}(\boldsymbol{\nu} \tilde{p})\left(\hat{P}^{\prime}\left|\bar{b}\langle-: \tilde{m} \| \tilde{c}\rangle^{\circ}\right| \bar{b}\langle-: \tilde{m} \| \tilde{c}\rangle_{i}^{\circ}\right) \equiv P^{\prime \prime}
$$

where $\hat{P} \stackrel{b(-: \tilde{m} \| \tilde{c})}{\longrightarrow}$ MIM $\hat{P}^{\prime}$

$$
\Downarrow
$$$$
P \equiv(\boldsymbol{\nu} \tilde{p})\left(\hat{P} \mid \bar{b}\langle-: \tilde{m} \| \tilde{c}\rangle_{i}^{\circ}\right) \stackrel{(i)}{\longmapsto}_{\text {MIM }}(\boldsymbol{\nu} \tilde{p})\left(\hat{P}^{\prime} \mid \bar{b}\langle-: \tilde{m} \| \tilde{c}\rangle_{i}^{\circ}\right) \equiv P^{\prime \prime \prime}
$$

$\stackrel{\mathrm{MTM}}{\sim}$

$\stackrel{\text { MTM }}{\sim}$

$Q \equiv(\boldsymbol{\nu} \tilde{q})\left(\hat{Q} \mid \bar{b}\left\langle-: \tilde{m}^{\prime} \| \tilde{c}\right\rangle_{i}^{\circ}\right) \stackrel{(i)}{\longmapsto}_{\text {MIM }}(\boldsymbol{\nu} \tilde{q})\left(\hat{Q}^{\prime} \mid \bar{b}\left\langle-: \tilde{m}^{\prime} \| \tilde{c}\right\rangle_{i}^{\circ}\right) \equiv Q^{\prime \prime \prime}$

where $\hat{Q} \stackrel{b\left(-: \tilde{m}^{\prime} \| \tilde{c}\right)}{\longrightarrow}$ мг $\hat{Q}^{\prime}$

$$
\begin{aligned}
& Q^{\prime} \equiv(\boldsymbol{\nu} \tilde{q})\left(\hat{Q}\left|\bar{b}\left\langle-: \tilde{m}^{\prime} \| \tilde{c}\right\rangle^{\circ}\right| \bar{b}\left\langle-: \tilde{m}^{\prime} \| \tilde{c}\right\rangle_{i}^{\circ}\right) \\
& \stackrel{(i)}{\longmapsto}_{\text {мтм }}(\boldsymbol{\nu} \tilde{q})\left(\hat{Q}^{\prime}\left|\bar{b}\left\langle-: \tilde{m}^{\prime} \| \tilde{c}\right\rangle^{\circ}\right| \bar{b}\left\langle-: \tilde{m}^{\prime} \| \tilde{c}\right\rangle_{i}^{\circ}\right) \equiv Q^{\prime \prime}
\end{aligned}
$$

Here, the format of $Q^{\prime \prime \prime}$ is a consequence of Lemma 4.2(2) by which $\hat{Q} \chi_{i}$, hence the replay move from $Q$ must originate from the unique output indexed by $i$. Now we conclude exactly as in the previous case.

- The move $\alpha$ is by process $\bar{b}\langle-: \tilde{m} \| \tilde{c}\rangle^{\circ}$ and is an output. Since the transition is observable we know that $b \in \mathbf{N}_{u}$ or $\circ=\varepsilon$ and, given that $P$ and $Q$ are compatible, we obtain that $\tilde{m}=\tilde{m}^{\prime}$. By Lemma 4.2(1) we also have that $i, b, \tilde{c}, \tilde{m} \in f n(P, Q)$. Thus:

$$
\begin{aligned}
& P^{\prime} \equiv(\boldsymbol{\nu} \tilde{p})\left(\hat{P}\left|\bar{b}\langle-: \tilde{m} \| \tilde{c}\rangle^{\circ}\right| \bar{b}\langle-: \tilde{m} \| \tilde{c}\rangle_{i}^{\circ}\right) \stackrel{\bar{b}\langle-: \tilde{m} \| \tilde{c}\rangle^{\circ}}{\longrightarrow}{ }_{\text {MIM }}(\nu \tilde{p})\left(\hat{P} \mid \bar{b}\langle-: \tilde{m} \| \tilde{c}\rangle_{i}^{\circ}\right) \equiv P \\
& Q^{\prime} \equiv(\boldsymbol{\nu} \tilde{q})\left(\hat{Q}\left|\bar{b}\langle-: \tilde{m} \| \tilde{c}\rangle^{\circ}\right| \bar{b}\langle-: \tilde{m} \| \tilde{c}\rangle_{i}^{\circ}\right) \stackrel{\bar{b}\langle-: \tilde{m} \| \tilde{c}\rangle^{\circ}}{\longrightarrow} \text { мІм }(\boldsymbol{\nu} \tilde{q})\left(\hat{Q} \mid \bar{b}\langle-: \tilde{m} \| \tilde{c}\rangle_{i}^{\circ}\right) \equiv Q
\end{aligned}
$$

In other words, we are back with $P$ and $Q$, which are MIM-bisimilar (and thus included in $\mathscr{R})$ by hypothesis.

- The move $\alpha$ is by process $\bar{b}\langle-: \tilde{m} \| \tilde{c}\rangle^{\circ}$ and is an intercepted output. We distinguish two further sub-cases. If the output, and its indexed copy are in clear, by the compatibility of $P$ and $Q$ we may conclude that $\tilde{m}=\tilde{m}^{\prime}=\tilde{c}$. Hence the transitions from $P^{\prime}$ and $Q^{\prime}$ are as follows:

$$
\begin{aligned}
P^{\prime} & \equiv(\boldsymbol{\nu} \tilde{p})\left(\hat{P}\left|\bar{b}\langle-: \tilde{m} \| \tilde{m}\rangle^{\circ}\right| \bar{b}\langle-: \tilde{m} \| \tilde{m}\rangle_{i}^{\circ}\right) \\
& \stackrel{(j) \dagger \bar{b}<-: \tilde{m} \| \tilde{m}\rangle_{j}^{\circ}}{\longmapsto} \underset{\text { MIM }}{ }(\boldsymbol{\nu} \tilde{p})\left(\hat{P}\left|\bar{b}\langle-: \tilde{m} \| \tilde{m}\rangle^{\circ}\right| \bar{b}\langle-: \tilde{m} \| \tilde{m}\rangle_{i}^{\circ} \mid \bar{b}\langle-: \tilde{m} \| \tilde{m}\rangle_{j}^{\circ}\right) \equiv P^{\prime \prime}
\end{aligned}
$$

and

$$
\begin{aligned}
Q^{\prime} \equiv & (\boldsymbol{\nu} \tilde{q})\left(\hat{Q}\left|\bar{b}\langle-: \tilde{m} \| \tilde{m}\rangle^{\circ}\right| \bar{b}\langle-: \tilde{m} \| \tilde{m}\rangle_{i}^{\circ}\right) \\
& \stackrel{(j)+\bar{b}\langle-: \tilde{m} \| \tilde{m}\rangle_{j}^{\circ}}{\longrightarrow \text { мтм }}(\boldsymbol{\nu} \tilde{q})\left(\hat{Q}\left|\bar{b}\langle-: \tilde{m} \| \tilde{c}\rangle^{\circ}\right| \bar{b}\langle-: \tilde{m} \| \tilde{c}\rangle_{i}^{\circ} \mid \bar{b}\langle-: \tilde{m} \| \tilde{c}\rangle_{j}^{\circ}\right) \equiv Q^{\prime \prime} .
\end{aligned}
$$

That $P^{\prime \prime} \stackrel{\text { MIM }}{\sim} Q^{\prime \prime}$ follows from our hypothesis $P \stackrel{\text { MTM }}{\sim} Q$, by Lemma $6.2(2)$. 
When $\circ=\bullet$, for $P^{\prime}$ we have:

$$
\begin{aligned}
& P^{\prime} \equiv(\boldsymbol{\nu} \tilde{p})\left(\hat{P}\left|\bar{b}\langle-: \tilde{m} \| \tilde{c}\rangle^{\bullet}\right| \bar{b}\langle-: \tilde{m} \| \tilde{c}\rangle_{i}^{\bullet}\right)
\end{aligned}
$$

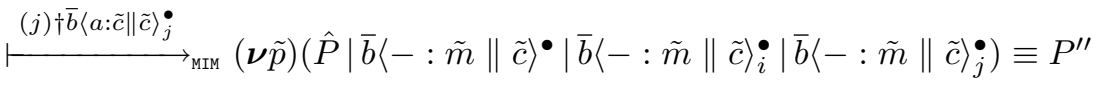

$$
\begin{aligned}
& Q^{\prime} \equiv(\boldsymbol{\nu} \tilde{q})\left(\hat{Q}\left|\bar{b}\left\langle-: \tilde{m}^{\prime} \| \tilde{c}\right\rangle \bullet\right| \bar{b}\left\langle-: \tilde{m}^{\prime} \| \tilde{c}\right\rangle_{i}^{\bullet}\right) \\
& \stackrel{(j) \dagger \bar{b}\langle-: \tilde{c} \| \tilde{c}\rangle_{j}^{\bullet}}{\longmapsto} \text { MIM }(\boldsymbol{\nu} \tilde{q})\left(\hat{Q}\left|\bar{b}\left\langle-: \tilde{m}^{\prime} \| \tilde{c}\right\rangle^{\bullet}\right| \bar{b}\left\langle-: \tilde{m}^{\prime} \| \tilde{c}\right\rangle_{i}^{\bullet} \mid \bar{b}\left\langle-: \tilde{m}^{\prime} \| \tilde{c}\right\rangle_{j}^{\bullet}\right) \equiv Q^{\prime \prime}
\end{aligned}
$$

That $P^{\prime \prime} \stackrel{\text { мim }}{\sim} Q^{\prime \prime}$ follows from our hypothesis $P \stackrel{\text { мim }}{\sim} Q$, again by Lemma $6.2(2)$.

There are no other moves, as there are no direct MIM-synchronizations for a trusted process. 


\section{Appendix B. Semantics of Eavesdroppers}

Reduction. Like for intercept, $\sigma$ is the substitution $\{b / z, \underline{a} / x, \tilde{p} / \tilde{y}, \tilde{c} / \tilde{w}\}$, and the $\tilde{p}$ are as follows: if $\circ=\bullet$ and $b \in \mathbf{N}_{t}$ then $\tilde{p}=\tilde{c}$ else $\tilde{p}=\tilde{m}$. Moreover, in the (Eavesdrop) rule, $i \notin\{b, \tilde{m}, \tilde{c}\}$.

(Eavesdrop Auth)

$\bar{b}\langle a: \tilde{m} \| \tilde{c}\rangle^{\circ}\left|? z(x: \tilde{y} \| \tilde{w})_{i}^{\circ} \cdot N \quad \longrightarrow \bar{b}\langle\underline{a}: \tilde{m} \| \tilde{c}\rangle^{\circ}\right|(\boldsymbol{\nu} i) N \sigma$

(Eavesdrop)

$\bar{b}\langle-: \tilde{m} \| \tilde{c}\rangle^{\circ}\left|? z(x: \tilde{y} \| \tilde{w})_{i}^{\circ} N \quad \longrightarrow \bar{b}\langle-: \tilde{m} \| \tilde{c}\rangle^{\circ}\right|(\boldsymbol{\nu} i)\left(\bar{b}\langle-: \tilde{m} \| \tilde{c}\rangle_{i}^{\circ} \mid N \sigma\right)$

\section{Labelled Transitions}

(Output Eavesdropped)

$\frac{b \notin \mathbf{N}_{t} \text { or } \circ \neq \bullet \quad i \notin\{b, \tilde{m}, \tilde{c}\}}{\bar{b}\langle-: \tilde{m} \| \tilde{c}\rangle^{\circ} \stackrel{(i) ? \bar{b}\langle-: \tilde{m} \| \tilde{c}\rangle_{i}^{\circ}}{\longrightarrow} \bar{b}\langle-: \tilde{m} \| \tilde{c}\rangle_{i}^{\circ} \mid \bar{b}\langle-: \tilde{m} \| \tilde{c}\rangle^{\circ}}$

(Output Eavesdropped Auth)

$b \notin \mathbf{N}_{t}$ or $\circ \neq \bullet \quad i \notin\{b, a, \tilde{m}, \tilde{c}\}$

$\bar{b}\langle a: \tilde{m} \| \tilde{c}\rangle^{\circ} \stackrel{(i) ? \bar{b}\langle a: \tilde{m} \| \tilde{c}\rangle_{i}^{\circ}}{\longrightarrow} \bar{b}\langle a: \tilde{m} \| \tilde{c}\rangle^{\circ}$

(Secret Output Eavesdropped)

$\frac{b \in \mathbf{N}_{t} \quad i \notin\{b, \tilde{m}, \tilde{c}\}}{\bar{b}\langle-: \tilde{m} \| \tilde{c}\rangle^{\bullet} \stackrel{(i) ? \bar{b}\langle-: \tilde{c} \| \tilde{c}\rangle_{i}^{\bullet}}{\longrightarrow} \bar{b}\langle-: \tilde{m} \| \tilde{c}\rangle_{i}^{\bullet} \mid \bar{b}\langle-: \tilde{m} \| \tilde{c}\rangle^{\bullet}}$

(Secret Output Eavesdropped Auth)

$$
b \in \mathbf{N}_{t} \quad i \notin\{b, a, \tilde{m}, \tilde{c}\}
$$

$\bar{b}\langle a: \tilde{m} \| \tilde{c}\rangle^{\bullet} \stackrel{(i) ? \bar{b}\langle a: \tilde{c} \| \tilde{c}\rangle_{i}^{\bullet}}{\longrightarrow} \bar{b}\langle a: \tilde{m} \| \tilde{c}\rangle^{\bullet}$

(Open Eavesdropped)

$\frac{N \stackrel{(\tilde{p}, i) ? \bar{b}\left\langle\underline{a}: \tilde{m} \|_{\tilde{c}}\right\rangle_{i}^{\circ}}{\longrightarrow} N^{\prime} \quad n \in\{b, \underline{a}, \tilde{m}, \tilde{c}\}-\{\tilde{p}, i\}}{(\boldsymbol{\nu} n) N \stackrel{(n, \tilde{p}, i) ? \bar{b}\langle\underline{a}: \tilde{m} \| \tilde{c}\rangle_{i}^{\circ}}{\longrightarrow} N^{\prime}} N^{\prime}$

(Synch Intercept)

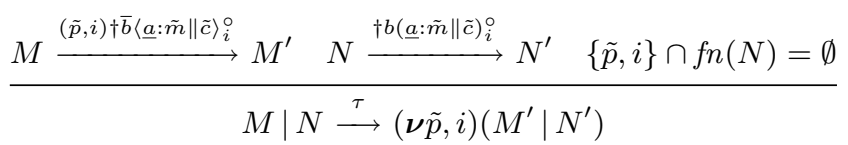

(Eavesdrop)

$? z(x: \tilde{y} \| \tilde{w})_{i} \cdot N \stackrel{? b(\underline{a}: \tilde{p} \| \tilde{c})_{i}^{\circ}}{\longrightarrow} N\{b / z, \underline{a} / x, \tilde{p} / \tilde{y}, \tilde{c} / \tilde{w}\}$ 